$\mathrm{UiO}$ : Department of Mathematics University of Oslo

\title{
Investigation of Free Surface Damping Models with Applications to Gap Resonance Problems
}

Kevin Markeng

Master's Thesis, Autumn 2015 



\begin{abstract}
Resonant behaviour in the gap between two adjacent bodies on a free surface is investigated numerically. The study is done within the framework of twodimensional potential theory. It is of common conception that traditional potential theory overpredicts the gap elevation around resonance due to neglect of viscous effects. In this study we consider empirical formulas for skin friction, eddy damping and bilge keel damping, and include the effects by a free surface damping model. A non-physical free surface damping model is also considered, and the damping effects from the two models are compared. Some brief comments are given on three-dimensional effects.
\end{abstract}





\section{Contents}

1 Introduction $\mathbf{2}$

1.1 Previous related studies . . . . . . . . . . . . . . . 2

1.2 Present study . . . . . . . . . . . . . . . . . . . . 3

1.2 .1 Scope ................... 3

2 Mathematical foundation $\mathbf{5}$

2.1 The boundary value problem .............. 5

2.2 The Green function and Green's theorem . . . . . . . . . . . . 7

3 The Gap Resonance Problem 9

3.1 Relevance ..................... . . . 9

3.2 Resonant behaviour . . . . . . . . . . . . . . . . . . . 9

3.3 Zeroth order mode; the piston mode . . . . . . . . . . . . . . . . 10

3.4 Potential Damping . . . . . . . . . . . . . . . . . . . 12

3.5 Viscous Damping Contributions . . . . . . . . . . . . . . . 12

3.5.1 Skin Friction damping . . . . . . . . . . . . . . . . . 14

3.5.2 Eddy damping . . . . . . . . . . . . . . . . . . 14

3.5.3 Bilge keel damping . . . . . . . . . . . . . . . . 15

4 Free Surface Damping Models 16

4.1 Introduction . . . . . . . . . . . . . . . . . . . 16

4.2 The Pressure Damping Model . . . . . . . . . . . . . . . . 16

4.2.1 Gap surface pressure distribution . . . . . . . . . . 17

4.2.2 Pressure generated potential . . . . . . . . . . . . 17

4.3 Newtonian Cooling . . . . . . . . . . . . . . . . . . 19

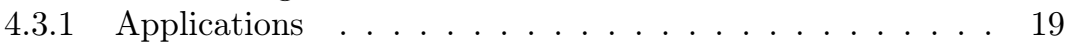

4.3.2 Formulation ................. 19

5 Numerical method and program structure $\quad 21$

5.1 Boundary element method . . . . . . . . . . . . . . . 21

5.1.1 System without additional damping . . . . . . . . . . 21

5.1.2 System with free surface damping . . . . . . . . . . 23

5.2 Program structure . . . . . . . . . . . . . . . 25

6 Numerical Analysis and Results $\quad 27$

6.1 Introductory comment . . . . . . . . . . . . . . . . . . . 27

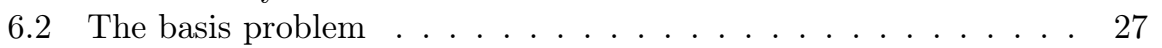

6.2.1 Forced heave motion . . . . . . . . . . . . . . 27

6.2.2 Incident wave upon rigid double-hull . . . . . . . . . . . . 31 
6.2.3 Incident wave upon freely floating body . . . . . . . . . 33

6.2.4 Comparison with 3D results from Wasim and comments

on three dimensional effects . . . . . . . . . . . . . 35

6.3 Free surface pressure model . . . . . . . . . . . . . . . . . . . 38

6.3 .1 Introductory remarks . . . . . . . . . . . . . . 38

6.3.2 Heaving of rectangular boxes . . . . . . . . . . . . . 39

6.4 Newtonian Cooling Damping Model _. . . . . . . . . . . . 45

6.4.1 Heaving of rectangular boxes . . . . . . . . . . . 45

6.5 Comparison of the damping models . . . . . . . . . . . . . 49

$\begin{array}{lll}7 & \text { Summary and conclusion } & 51\end{array}$

$\begin{array}{ll}\text { Appendices } & 52\end{array}$

A Greens theorem $\quad 53$

B Equivalent linearization $\quad 54$

C Convergence test $\quad 55$

D Equation of motion $\quad 56$ 


\section{Forord}

Jeg ønsker å takke mine veiledere, professor John Grue og Dr. Scient Torgeir Vada for oppfølging og veiledning i forbindelse med oppgaven. John Grue introduserte meg til fagfeltet hydrodynamikk, og hans engasjement og kunnskap har vært en inspirasjonskilde gjennom hele studiet. Samarbeidet med Torgeir Vada (Product Manager ved DNV GL Software) har ikke bare vært lærerikt, men også svært oppmuntrende.

Min familie og kjæreste har vist stor interesse og støttet meg gjennom hele studiet, og for dette er jeg høyst takknemlig.

Jeg vil takke alle på kontoret hos DNV GL for å ha latt meg gjeste kontoret, og for å ha gitt meg et innblikk i deres virksomhet. Spesielt takk til Peter, Kaija og Styrk som har vært behjelpelige gjennom hele prosessen.

En takk til Trond Svandal for mange gode diskusjoner rundt oppgavens tema. Til mine studiekamerater på lesesalen i 9. etasje ønsker jeg å takke for godt samhold og god stemning, på tross av at quiz-laget vårt aldri ble en stor suksess. 



\section{Chapter 1}

\section{Introduction}

When two or more structures are located in vicinity of each other, large resonant motion may occure in the narrow gap between them. This may also be the case if a single body has some sort of opening, for instance a moonpool. Gap surface response and hydrodynamic coefficients has proven to be a challenging task to calculate accuratly for this particular problem.

Seakeeping softwares based on the Boundary Element Method (BEM) is still the most popular engineering tool for analysing wave loads on marine structures. This is mainly due to high computer efficiency and the simplicity of defining grid points. However, traditional BEM solvers does not take viscous effects into account. In certain applications, like resonant behaviour in narrow gaps or moonpools, it has been demonstrated that viscous damping is important and should not be neglected. The general conception is that the results acquired from BEM solvers overpredict the fluid motion in the gap due to the neglection of viscosity. The overpredicted fluid motion is also reflected in the pressure forces and may result in misleading conceptions of the situation.

Viscous effects are best captured by Computational Fluid Dynamics (CFD) programs which solves the Navier Stokes-equation. The grid required for analysing wave-body interaction within such a framework leads to computer heavy analysis and high CPU time. Methods for including the effects of viscosity in the more effective BEM environment is therefore sought after.

\subsection{Previous related studies}

Many studies are devoted to gap resonance problems as it is relevant within marine activity. Molin [9] has provided an analytical expression for the location of the gap resonance frequency between rectangular boxes. However, to get amplitudes and transfer functions, the full problem must be solved numerically. Sun [14] analysized both the first order and the second order problem with three dimensionsional potential theory. The non-linear effects were small, except for in some special cases in context with higher order resonance frequencies. Kristiansen and Faltinsen [7] solved the non-linear potential problem in two dimensions, and applied an inviscid vortex tracking method in the BEMformulation. The results were compared to model tests. They reported small second order effects, and that the measured gap amplitude from the model test 
were $1 / 3$ of the calculated amplitude. They concluded that flow separation at the bilges were the main reason for the discrepancy, and managed to get better agreement with the use of the vortex tracking method. To provide external damping, a so called damping lid may be placed at the free surface in the gap or moonpool. Buchner et al. [2] used a rigid lid with a tunable damping effect, while a flexible lid was provided by Newman [11]. A free surface damping method was provided by Chen [3]. He introduced what is referred to as "potential flow of fairly perfect fluid" into the BEM formulation. In his formulation the provided damping is proportional to the velocity, but of opposite sign. The damping term is also proportional to a damping coefficient. The damping effect must be tuned to the results match those from model tests.

Recently, Kristiansen and Faltinsen [8] used a domain-decomposition method to study forced heave of a twin hull. The Navier-Stokes formulation was applied in a domain close to the body while the rest of the fluid were treated by linear potential theory. Their computed surface elevation amplitude matched very well with experimental data. The method was reported as promising as it captures the important viscous effects without use of empirical input, and it is significantly faster than a full Navier-Stokes solver.

\subsection{Present study}

We will investigate methods to correct the linearly overpredicted fluid motion at resconance frequencies in narrow gap problems. In particular we are focusing on the wave response occuring at the first resonance frequency, later referred to as the piston mode response. The study is done within the frames of BEM, as this is the current industry standard for wave-body analysis. We restrict ourselves to a two-dimensional approach for practical purposes. We choose to focus our attention on rigidly connected rectangular barges separated by a gap, as displayed in figure 1.1. In the BEM-formulation it is convinient to introduce damping in the free surface boundary condition. It is essential that the applied damping is related to the actual physical viscous damping. Attention will be given to energy dissipation. We use empirical formulas with strong foundation to estimate viscous forces. Two different free surface damping methods will be investigated and compared. They are referred to as the Pressure Damping Model, and the Newtonian Cooling Damping Model.

\subsubsection{Scope}

- Identify important aspects to gap resonance problems

- Give a general formulation of the Pressure Damping Model

- Find models for the damping due to viscous effects, and relate them to the Pressure Damping Model

- Implement and investigate the Newtonian Cooling Damping Model, currently used in HydroD Wasim

- Compare the two damping models 
- Compare two-dimensional and three-dimensional computations. HydroD Wasim will be used for three-dimensional computations

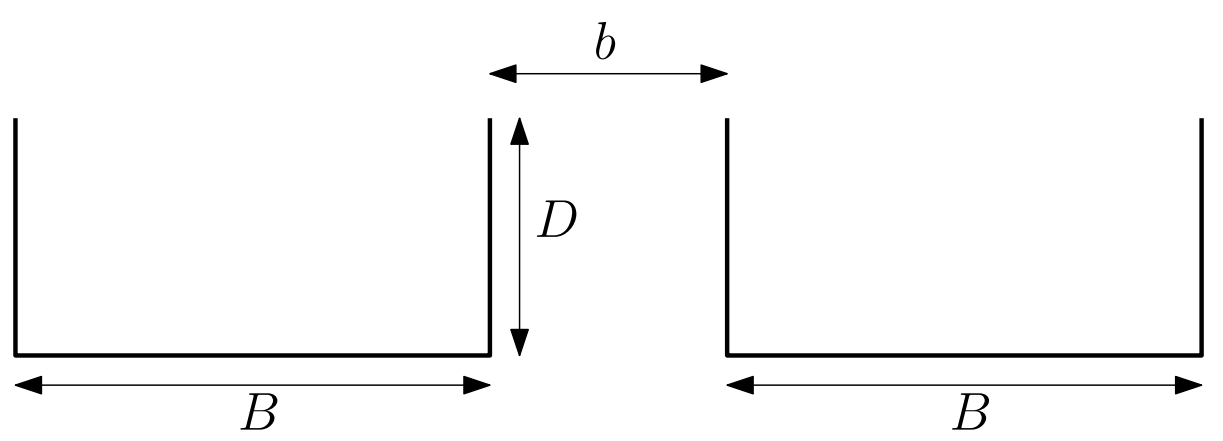

Figure 1.1: Two closely separated hulls. Each hull has a width $B$ and draft $D$, and are separated by $b$. In this study the hulls are considered to be rigidly connected. This could be interpreted as the cross section of a barge with an infinitely long moonpool. Throughout the thesis we will refere to the opening as the gap. 


\section{Chapter 2}

\section{Mathematical foundation}

\subsection{The boundary value problem}

We consider a plane progressive wave of small amplitude interacting with a floating rigid body. The body will then undergo simple harmonic oscillations described by the body displacement from equilibrium . We assume irrotational and incompressible flow, and apply linear potential theory. The velocity potential $\Phi$ satisfies the Laplace equation in the fluid domain

$$
\nabla^{2} \Phi=0 .
$$

All dynamic quantities are assumed to be harmonic in time, such that $\Phi$ may be expressed as

$$
\Phi=\operatorname{Re}\left\{\varphi e^{i \omega t}\right\}
$$

where Re denotes the real part and $\omega$ is the frequency of the incident wave. Similarly, all other quantities derived from $\varphi$ is understood to be a complex amplitude and the physical values is the real part of the product between the complex amplitude and the time factor $e^{i \omega t}$. We denote the free surface elevation as $\zeta$, and define it as

$$
\zeta=\operatorname{Re}\left\{\eta e^{i \omega t}\right\} .
$$

Two conditions apply on the free surface, these are the kinematic and the $d y$ namic boundary conditions. The kinematic boundary condition requires the fluid velocity at the free surface to be the same as the surface velocity itself,

$$
\frac{\partial \zeta}{\partial t}=\frac{\partial \Phi}{\partial z}
$$

The linear dynamic condition follows from the linearized Bernoulli equation,

$$
\frac{\partial \Phi}{\partial t}+g z=-\frac{1}{\rho} p
$$

by stating that the gauge pressure $p$ is zero at the free surface and evaluating at $z=\zeta$, 


$$
\frac{\partial \Phi}{\partial t}+g \zeta=0
$$

The kinematic and dynamic boundary condition may be combined by differentiating (2.6) with respect to $t$ and substitute (2.4), to form a single free surface boundary condition for $\Phi$,

$$
\frac{\partial^{2} \Phi}{\partial t^{2}}+\frac{\partial \Phi}{\partial z}=0
$$

By taking the time derivative and remove the time factor we may express the boundary condition in terms of the time independent potential $\varphi$,

$$
-\frac{\omega^{2}}{g} \varphi+\frac{\partial \varphi}{\partial z}=0
$$

The fluid velocity at the body boundary follows the same velocity as the boundary itself,

$$
\frac{\partial \Phi}{\partial n}=\mathbf{U} \cdot \mathbf{n}+\boldsymbol{\Omega} \cdot(\mathbf{r} \times \mathbf{n}),
$$

where $\mathbf{n}$ is the normal vector, $\mathbf{r}$ is the radius vector from center of rotation, $\mathbf{U}$ and $\boldsymbol{\Omega}$ are the body translational velocity and the body angular velocity respectively. If six degrees of freedom in three dimensions are considered, the components of the body velocity are

$$
U_{j}=\operatorname{Re}\left\{i \omega \xi_{j} e^{i \omega t}\right\} \quad j=1,2, \ldots, 6
$$

where $\xi_{j}$ is the complex amplitude, and $j=1,2, \ldots, 6$ is surge, sway, heave, roll, pitch and yaw respectively. In two dimensions the relevant degrees of freedom are $j=2,3,4$.

The velocity potential may be divided into a radiation potential and diffraction potential, and summed by the super position principle

$$
\Phi=\operatorname{Re}\left\{\left(\varphi_{R}+\varphi_{D}\right) e^{i \omega t}\right\} .
$$

The radiation potential is due to the motion of the body while the diffraction potential represents the incident wave and the disturbance due to the presence of the body. The radiation potential is the sum of contributions from motions in each degree of freedom. For motion in three dimensions we have

$$
\varphi_{R}=\sum_{j=1}^{6} \xi_{j} \varphi_{j}, \quad j=1,2, \ldots, 6
$$

where $\xi_{j}$ is the complex displacement amplitude. The body boundary condition for the radiation potentials follows from (2.9), 


$$
\begin{array}{rlrl}
\frac{\partial \varphi_{j}}{\partial n} & =i \omega n_{j} & j & =1,2,3 \\
\frac{\partial \varphi_{j}}{\partial n} & =i \omega(\mathbf{r} \times \mathbf{n})_{j-3} & j & =4,5,6 .
\end{array}
$$

The diffraction potential consists of the incident wave potential $\varphi_{0}$ with amplitude $A$, and the scattering wave potential $\varphi_{s}$ which is due to the presence of the fixed body

$$
\varphi_{D}=A\left(\varphi_{0}+\varphi_{s}\right),
$$

where $\varphi_{0}$ is on the form

$$
\varphi_{0}=\frac{i g}{\omega} e^{k y-i k x} .
$$

As the body is considered fixed with respect to $\varphi_{D}$, the right hand side of (2.9) is zero. It follows that

$$
\frac{\partial \varphi_{s}}{\partial n}=-\frac{\partial \varphi_{0}}{\partial n} .
$$

As $\varphi_{j}$ and $\varphi_{s}$ is a consequence of the presence of the body, we must require that the associated waves radiates outwards from the body. This is the radiation condition, and is necessary for uniqueness of the boundary value problem. In three dimensions we require

$$
\varphi_{j} \propto R^{-1 / 2} e^{-i k R}, \quad \text { as } \mathrm{R} \rightarrow \infty \quad j=1,2, \ldots, 6, s
$$

where $\mathrm{R}$ is the radial distance from the body. The two dimensional radiation condition is

$$
\varphi_{j} \propto e^{\mp i k x}, \quad \text { as } \mathrm{x} \rightarrow \pm \infty \quad j=2,3,4, s .
$$

The boundary value problem defined by (2.1), (2.8), (2.13) and (2.16) will be referred to as the basis problem.

\subsection{The Green function and Green's theorem}

Details on the following is presented in Appendix A while a brief introduction is presented in this section. Green's theorem may be utilized to form integral equations for unknown potentials. We introduce the Green function for infinite depth in two dimensions [16],

$$
\begin{aligned}
G(\mathbf{x}, \boldsymbol{\xi})= & \ln r_{1}-\ln r_{2}-2 P V \int_{0}^{\infty} \frac{1}{k-\nu} e^{k(z+\zeta)} \cos (k(x-\xi)) \mathrm{d} k \\
& +2 \pi i e^{\nu(z+\zeta)} \cos (\nu(x-\xi)),
\end{aligned}
$$


where $r_{1}=\sqrt{(x-\xi)^{2}+(z-\zeta)^{2}}, r_{2}=\sqrt{(x-\xi)^{2}+(z+\zeta)^{2}}$ and $\mathbf{x}=(x, z)$, $\boldsymbol{\xi}=(\xi, \zeta)$ are understood to be coordinates in the two-dimensional plane. The Green function (2.19) represents a pulsating source potential at point $\boldsymbol{\xi}$, evaluated at point $\mathbf{x}$, and satisfies the free surface boundary condition (2.8) and the radiation condition (2.18). A fluid domain is illustrated in figure 2.1. Applying Green's theorem on the fluid domain to a unknown potential $\varphi$ and $G$ yields an integral equation for $\varphi$,

$$
\int_{S}\left(\varphi(\boldsymbol{\xi}) \frac{\partial G(\boldsymbol{\xi} ; \mathbf{x})}{\partial n_{\boldsymbol{\xi}}}-G(\boldsymbol{\xi} ; \mathbf{x}) \frac{\partial \varphi(\boldsymbol{\xi})}{\partial n_{\xi}}\right) \mathrm{d} \boldsymbol{\xi}= \begin{cases}0, & \text { outside the fluid domain } \\ \pi \varphi(\mathbf{x}), & \text { on the boundary } \\ 2 \pi \varphi(\mathbf{x}), & \text { inside the fluid domain }\end{cases}
$$

The fact that (2.19) satisfy the free surface boundary conditions reduce the integral to be taken only over the body surface. This is an essential feature of the Green function. Equation (A.4) may be used to form integral equations for the unknown radiation and scattering potentials. Then $\frac{\partial \varphi}{\partial n}$ is known from the boundary conditions, and the equation is typically organized as

$$
\int_{S_{w}} \varphi(\boldsymbol{\xi}) \frac{\partial G(\boldsymbol{\xi} ; \mathbf{x})}{\partial n_{\boldsymbol{\xi}}} \mathrm{d} \boldsymbol{\xi}-\left(\begin{array}{c}
\pi \\
2 \pi
\end{array}\right) \varphi(\mathbf{x})=\int_{S_{w}} G(\boldsymbol{\xi} ; \mathbf{x}) \frac{\partial \varphi(\boldsymbol{\xi})}{\partial n_{\boldsymbol{\xi}}} \mathrm{d} \boldsymbol{\xi} .
$$

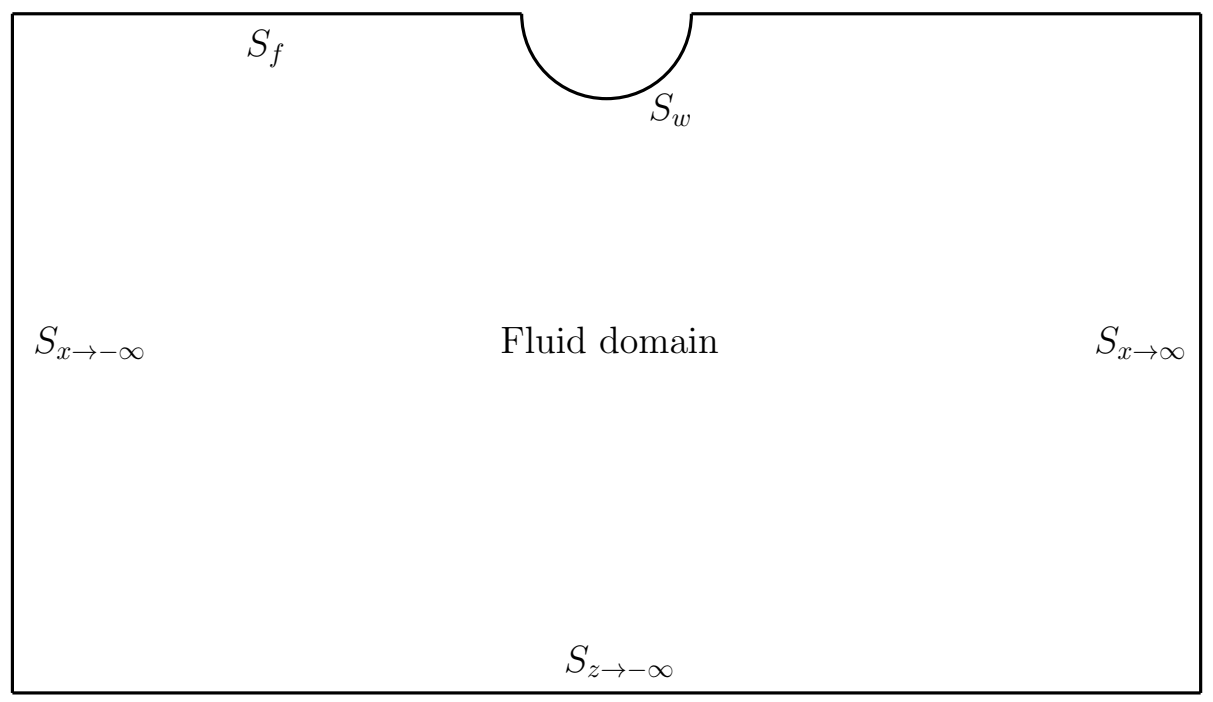

Figure 2.1: Illustration of the fluid domain with boundary $S=S_{f}+S_{w}+S_{x \rightarrow \infty}+$ $S_{x \rightarrow-\infty}+S_{z \rightarrow-\infty}$. The free surface is denoted by $S_{f}$, and $S_{w}$ represents a rigid body. 


\section{Chapter 3}

\section{The Gap Resonance Problem}

\subsection{Relevance}

The topic of this study applies to various marine applications. Gap resonance behaviour may occure in situations where one or several structures bounds the free surface in any manner. Some of the most important examples from the maritime industry beeing ships in side-by-side configuration, ship besides terminals relevant to on- and offloading operations, constructions with moonpools and multi-hull vessels.

\subsection{Resonant behaviour}

When a structure encloses a part of the free surface, this enclosed free surface will be subject to violent motions at some frequencies of oscillation. We will refere to the enclosed portion of the free surface as the gap surface. Motion of the gap surface may be trigged in three conceptual different scenarios. If the structure is fixed, the gap surface motion can be trigged by an incident wave. Similarly, gap surface motion occurs if the body is forced to oscillate. We will first discuss these two cases, as they share some important similarities and gives importent insight to the problem. Regardless if the gap surface oscillation is caused by incident wave or forced body motion, there exists an infinite number of what we will refere to as gap resonance frequencies. The gap surface will at these frequencies undergo large motions, and the shape, or mode, of the surface will vary for each gap resonance frequency. The location of these frequencies are only dependent on the geometrical shape of the body. The third case is when the body is free to respond to an incident wave field. Interestingly, the gap surface response at the gap resonant frequency is very small in this case. However, large elevation occurs when the structure itself undergo large motions. We will refere to the frequencies where peak structure response occurs as body resonance frequencies. It will later be clear that the structure response is indeed dependent on the gap resonance frequency. 


\subsection{Zeroth order mode; the piston mode}

As discussed, each gap resonance frequency has its associated gap surface mode. There is one particular mode which in many ways differs from the others. This is the first mode occuring at the first gap resonance frequency, often referred to as the zeroth mode or the piston mode. At this mode, the gap surface is oscillating without variations across the gap. That is, the fluid in the gap is moving with approximately uniform velocity and acts almost like a rigid body. As an counterexample, this resonant behaviour differs from that one might experience in a closed container. Linear resonant motion in a closed tank will consist of purely antisymmetric sloshing modes due to mass conservation in the tank. In the gap resonance problem the partially enclosed fluid is free to interact, or communicate, with the rest of the fluid domain. See figure 3.1. This communication is an important aspect of the phenomenon.

The piston mode will not necessarily be the first resonant mode encountered. For example, if a rigid body with a gap is forced to oscillate in sway, one should expect that the first encountered resonant behaviour is at the second or third resonance frequency. The first resonance occuring in forced heave will always be the piston mode resonance. Examples of sway and heave induced motion are shown in figure 3.2 and 3.3 .

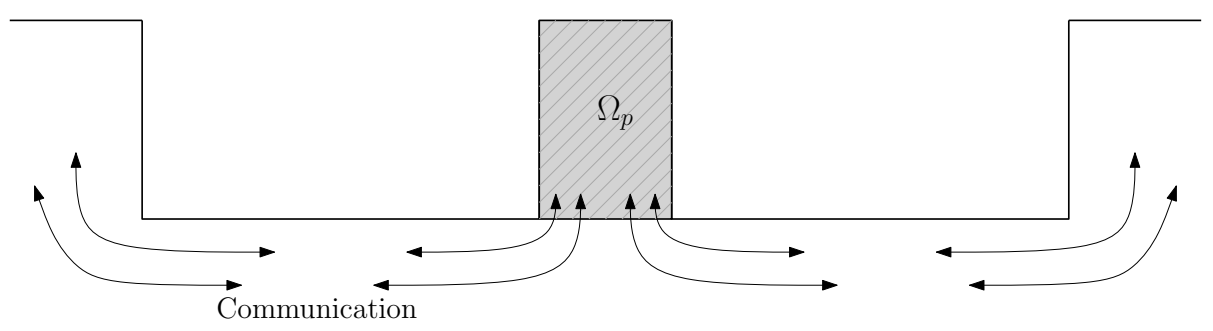

Figure 3.1: The "Piston body", or the partially enclosed fluid, is indicated by the shaded area and denoted by $\Omega_{p}$. The important communication between the partially enclosed fluid and the fluid outside the gap is indicated by the arrows. 


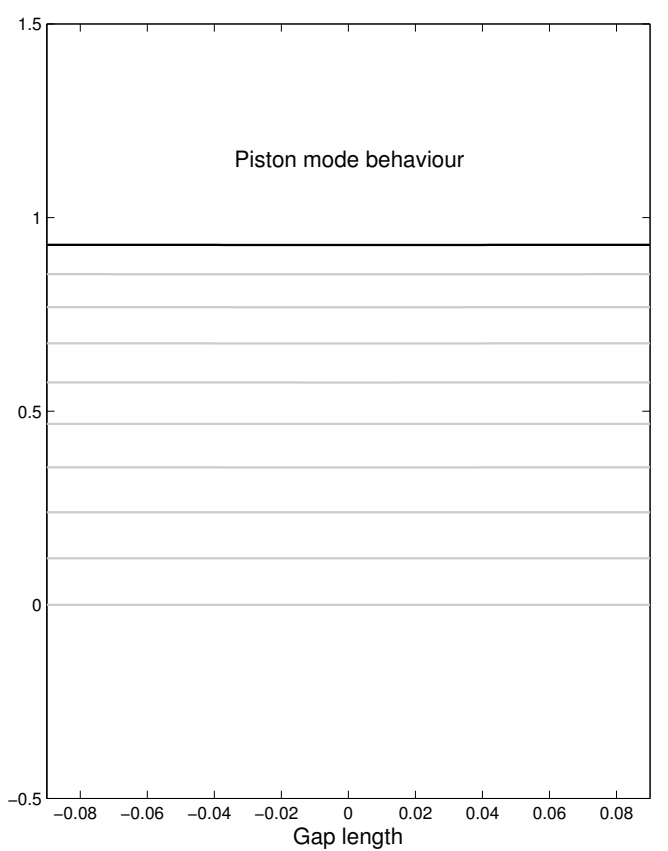

Figure 3.2: Example of heave induced piston mode behaviour. The surface elevation is displayed for a quarter of a periode, with grey lines indicating previous time steps.

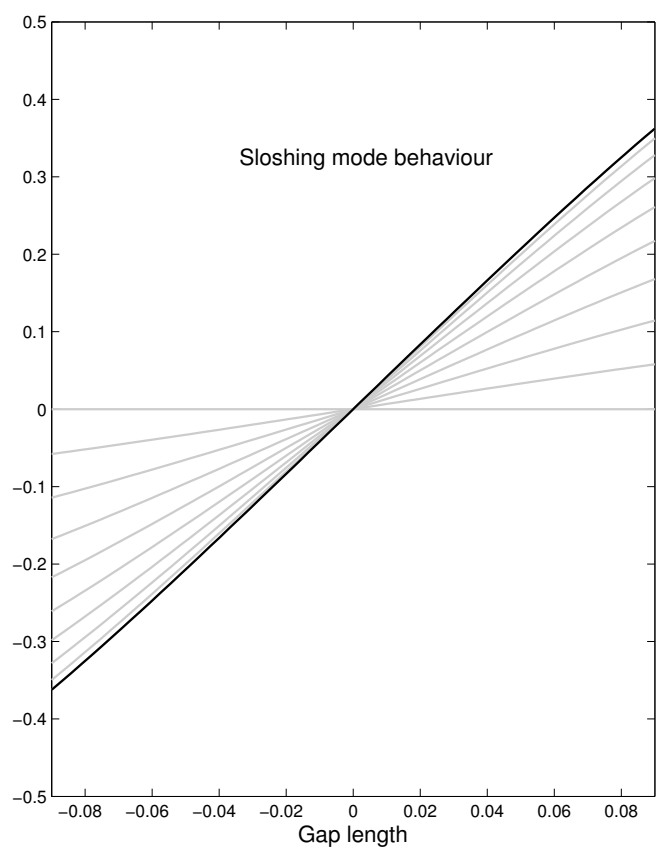

Figure 3.3: Example of sway induced sloshing behaviour. The surface elevation is displayed for a quarter of a periode, with grey lines indicating previous time steps. 


\subsection{Potential Damping}

Potential damping is associated with outgoing waves. When the resonant piston mode elevation in the gap is trigged at the gap resonance frequency, the amplitude of oscillation will stay finite. This is a consequence of the communiction between the fluid in the gap and the external fluid. Energy will be trasported away from the structure with the outgoing waves. The generation of waves is captured by potential theory, and it is the sole source of damping if external damping mechanisms are not introduced.

\subsection{Viscous Damping Contributions}

In many applications in marine hydrodynamics, viscous effects are considered to be small, and therefore potential theory based codes proves to be a powerful tool. However, experiments suggests that this is not the case for the narrow gap problem [7]. When potential calculations are compared to experimental data it shows clear tendencies that the potential calculations overpredict fluid velocities. Many numerical studies have investigated the effect of non-linear potential damping with the conclusion that these effects are negligible for the piston mode motion [7][14]. It is of common conception that the discrepancy between potential solutions and experimental data is due to the lack of viscous damping in the potential calculations. Viscous damping is considered to be of non-linear character. As gap surface motion may become very large it is reasonable to expect viscous damping to be a considerable contribution to the total damping. The viscous forces in play will in general dissipate energy, and hence both gap surface elevation and body motions should be reduced. Accurate numerical calculations of the viscous effects can be demanding, and from a practical point of view not feasible for large marine structures. Therefore, empirical formulas which approximates viscous effects is of great value.

In context within marine hydrodynamics where viscous effects are of importance, one often seek to estimate the viscous drag force on moving bodies. In the present study, the main interest is the viscous damping of the piston-mode elevation. However, if a viscous drag force is acting on the body, a equal but opposite force is inflicted on the fluid.

A quite general expression for the viscous force acting on a body ocillating in unbounded fluid is given by

$$
F_{D}=\frac{1}{2} \rho L^{2} C_{D}|v| v
$$

where $\rho$ is the density of the fluid, $v$ the relative velocity between the body and the fluid, $L$ is a characteristic length and $C_{D}$ is a problem specific drag coefficient. The drag coefficient will typically be a function of the Reynolds number defined as

$$
R e=\frac{v L}{\nu},
$$

where $\nu$ is the kinematic viscosity. The drag coefficient have to be empirically determined. A number of experimental studies are devoted to this purpose, and reliable values for the drag coefficient is available for various applications. 
As noted, (3.1) is based on the assumption that the fluid is unbounded. In our problem, a free surface is present. Nevertheless, we assume that the presence of the free surface has small effects on the viscous forces. Such an assumption is not unusual. Equation (3.1) is often used to estimate viscous forces on jackets and risers, and roll damping of ships or other structures where a free surface is indeed present.

We will consider three different contributions to the viscous force acting on the piston behaved fluid motion in the gap. These are the effect of skin friction, vortex shedding at the bilge, also reffered to as eddy making damping, and additonal effect if bilge keels are present. Figure 3.4, 3.5 and 3.6 illustrates the different effects. We may then express our total viscous force as

$$
F_{\text {viscous }}=F_{s}+F_{e}+F_{b k},
$$

where $F_{s}, F_{e}, F_{b k}$ is the viscous force contribution due to skin friction, eddy making and bilge keels, respectively. The three components will be further discussed in the following.
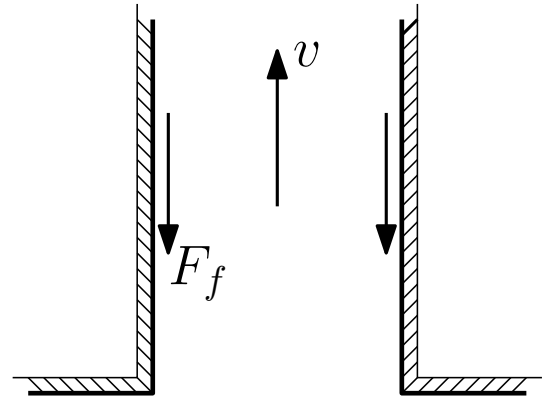

Figure 3.4: Illustration of friction force in the gap.

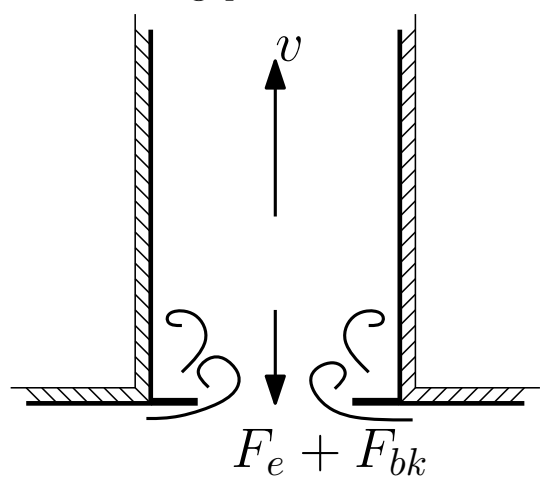

Figure 3.6: Illustration of structure with bilge keels. The presence of bilge keels will typically intensify the vortex shedding.

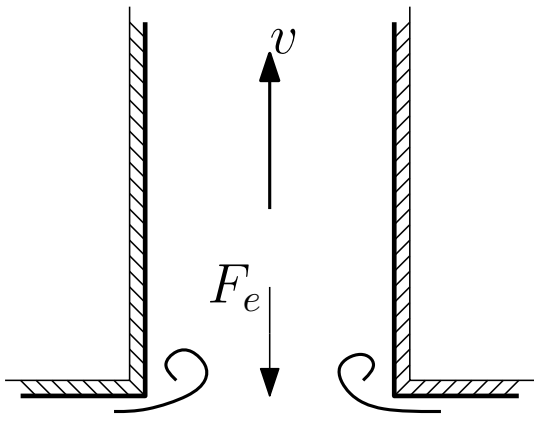

Figure 3.5: Illustration of vortex shedding at the bilge corners. 


\subsubsection{Skin Friction damping}

When fluid flows over the body surface it gives rise to skin friction stress. The reason for such a stress is the internal forces acting between particles. This viscous effect occur mainly in immediate vicinity of the body surface. The effect of the stress on the fluid is a tangential friction force in the opposit direction of the relative velocity between the fluid and the body surface. The friction force on a infinitesimal part of the body is gives as

$$
\mathrm{d} F=-\frac{1}{2} \rho C_{F}|v| v \mathrm{~d} S
$$

where $C_{F}$ is a friction drag coefficient. We only consider friction force on the part of the wetted surface which enclose the narrow gap. This is the inner side of the hulls which we denote $S_{w_{g}}$. The friction force elsewhere will presumably be negligible because velocities will be relatively small. The total friction force (per unit depth) is now defined by the integral

$$
F_{f}=-\frac{\rho}{2} C_{F} \int_{S_{w_{g}}}|v| v \mathrm{~d} S .
$$

Under the piston mode assumption, $v$ is not a function of space and may be moved outside the integral sign. We focus our attention on rectangular hulls, and the integral is evaluated over the draft of the body which define the gap

$$
F_{f}=-\rho C_{f} D|v| v
$$

Based on experiments with flow over flat plates, drag coefficients as function of the Reynolds number and plate dimensions has been proposed by Shoenherr (e.g. see Newman 1977, ch. 2.5). In our numerical study in Chapter 6 the relevant value for the friction drag coefficient is $C_{F} \approx 0.005$.

\subsubsection{Eddy damping}

Eddy damping is the effect associated with vortex shedding at the bilge. This issue is highly relevant in context of roll damping, and it is also speculated that this is a major contribution to damping of large piston mode motion in narrow gap problems. Berman [1] has suggested values for the drag coefficient related to vortex shedding based on experiments with cylinders of different shapes. In his experiments the geometries were fully submerged. The reported findings is listed here as

$$
\begin{aligned}
& C_{e} \approx 8.0 \mathrm{KC}^{-1 / 3} \quad \text { (flat plate) } \\
& C_{e} \approx 5.0 \quad \text { (diagonal square) } \\
& C_{e} \approx 3.0 \quad \text { (facing square) } \\
& C_{e} \approx 0.2 \mathrm{KC} \quad \text { (circular cylinder) }
\end{aligned}
$$

where $\mathrm{KC}$ is the Keulegan-Carpenter number defined as 


$$
\mathrm{KC}=\frac{V_{\max } T}{L_{c}}
$$

where $T$ is the period of oscillation and $U_{\max }$ is the maximum velocity.

To the present study, the coefficient for diagonal square is relevant. To make use of the values for $C_{e}$ in (3.7), some assumptions have to be made. We assume that the vortex shedding from the bilge corners is a local effect, such that the bilge corners may be regarded as fully submerged. We also assume that the effect of the two corners facing the gap is equivalent to $1 / 2$ of the effect of the facing square in (3.7), with the width of the square as the characteristic length. This means that we regard the vortex shedding effect from each corner in (3.7) as equal. These may be regarded as quite rough estimates, but nevertheless we hope to capture the leading effect. The expression for the eddy making force (per unit depth) is then given by

$$
F_{e}=-\frac{1}{2} \rho B \frac{C_{e}}{2}|v| v
$$

with $C_{e}=3.0$.

\subsubsection{Bilge keel damping}

Ships are often equipped with bilge keels to reduce roll response. The bilge keels introduce viscous damping mainly due to vortex shedding. When these keels are interacting with the large fluid motion in narrow gap, it should be expected that the viscous effects from the keels is an important damping contribution of the piston-like fluid motion. We consider two bilge keels as in figure 3.6, each with width $B_{b k}$. We apply the flat plate drag coefficient from (3.7), and do the assumption that the effect of the two keels, each with width $B_{b k}$ and one end pointed out in the stream, equals the effect of a flat plate with width $2 B_{b k}$ with two open ends. The viscous force from the bilge keels on the fluid (per unit depth) is expressed as

$$
F_{b k}=-\frac{1}{2} \rho C_{e} 2 B_{b k}|v| v,
$$

with $C_{e}=8.0 \mathrm{KC}^{-1 / 3}$. 


\section{Chapter 4}

\section{Free Surface Damping Models}

\subsection{Introduction}

The energy will always be conserved in the traditional potential formulation. This is an unphysical characteristics of the potential formulation, but it is often rightfully justified by assuming that dissipative effects are small. However, energy will dissipate if the fluid is viscous. It is stated that viscous effects should not be neglected if a narrow gap is present [5][7]. The forces due to viscous effects were discussed in chapter 3 , and it was indicated that they will result in decreased free surface elevation in the gap. In this chapter we formulate two methods which provide additional damping of the free surface elevation. They are referred to as the Pressure Damping Model and the Newtonian Cooling damping model. The methods are within the framework of potential theory, and external damping is introduced in the free surface boundary condition. The Pressure Damping Model will be directly related to the viscous forces considered in chapter 3, while the other method is of empirical character. Both methods will dissipate energy from the system.

\subsection{The Pressure Damping Model}

The Pressure Surface method has been used to study the OWC (Oscillating Water Column) wave energy devices, and surface effect ships [13][15]. In those cases, a pressure due to fans or compressed air will be distributed on an area of the free surface. We will utilize this method and relate the pressure force to the viscous forces analyzed in chapter 3 . It is important to emphasize that no physical pressure is acting on the gap surface in our problem. However, when the gap is narrow we assume that viscous forces acting in the gap will have an approximatly uniform damping effect on the piston-like fluid response. 


\subsubsection{Gap surface pressure distribution}

In Chapter 3 we considered viscous forces in the gap on the general form $F_{\text {viscous }}=-\frac{1}{2} \rho L_{c}^{2} C_{D}|v| v$. We assume that the effect on the piston mode motion in the gap will be uniform and expresses the force as a pressure distribution on the gap surface,

$$
P=-\frac{F_{\text {viscous }}}{b}
$$

where $b$ is the gap length from figure 1.1. The pressure will now be on the general form

$$
P=\frac{1}{2} \rho \frac{L_{c}^{2}}{b} C_{D}|v| v
$$

The expression may be linearized by the method of equivalent linearization. Details on the procedure is given in Appendix B. (4.2) can then be expressed as

$$
P \approx \frac{4}{3 \pi} \rho C_{D} V_{0} \frac{L_{c}^{2}}{b} v
$$

It is important to note that expression (4.3) is still quadratic in amplitude. As we assume the fluid velocity $v$ to be uniform in the gap, the velocity is related to the potential and the elevation through (2.4),

$$
v=\frac{\partial \Phi}{\partial z}=\frac{\partial \zeta}{\partial t}
$$

$P$ may then be expressed as

$$
P=\operatorname{Re}\left\{p e^{i \omega t}\right\}=\operatorname{Re}\left\{\frac{4}{3 \pi} \rho C_{D}|i \omega \eta| \frac{L_{c}^{2}}{b} i \omega \eta e^{i \omega t}\right\}
$$

The velocity is taken to be the velocity from the basis problem, without any additional free surface damping.

Note: By the numerical study on forced heave motion conducted in section 6.3, we have reached the conclusion that the pressure should have the phase of the body motion rather than the fluid gap velocity to get the desired effect. See the discussion in section 6.3.1.

\subsubsection{Pressure generated potential}

When pressure is acting on the free surface, the following boundary condition apply

$$
-\frac{\omega^{2}}{g} \varphi_{p}+\frac{\partial \varphi_{p}}{\partial z}=-\frac{i \omega}{\rho g} p,
$$


where $\varphi_{p}$ is the pressure generated potential. The pressure is introduced on the gap surface. The total potential (2.11) is thereby extended to include the velocity potentials due to free surface pressure in the gap

$$
\Phi=\operatorname{Re}\left\{\left(\varphi_{R}+\varphi_{D}+\varphi_{p}\right) e^{i \omega t}\right\},
$$

where $\varphi_{p}$ is the pressure generated potential. The introduction of the pressure generated potentials requires additional boundary conditions to the original boundary value problem. On the wetted surface we have

$$
\frac{\varphi_{p}}{\partial n}=0 .
$$

On the gap surface the pressure generated potential fulfill (4.6) while (2.8) holds for the potentials generated from body motion and scattering.

The field equation (2.1) and the free surface condition outside the body (2.8) is unchanged. Note that for the potentials generated from motion of the body, as well as for the diffraction potential, the boundary value problem is unchanged. These potentials can be solved separately by $(2.21)$ without any additions. For the pressure generated potential and its boundary conditions some modifications to the integral equations are required. The Green function does not satisfy the gap surface condition for $\varphi_{p}$ and thus the integral does not vanish as in (2.21). The integral is extended to include the gap surface $S_{g}$,

$$
\left(\begin{array}{c}
\pi \\
2 \pi
\end{array}\right) \varphi_{p}(\mathbf{x})=\int_{S_{w}+S_{g}}\left(\varphi_{p}(\boldsymbol{\xi}) \frac{\partial G(\boldsymbol{\xi} ; \mathbf{x})}{\partial n_{\boldsymbol{\xi}}}-G(\boldsymbol{\xi} ; \mathbf{x}) \frac{\partial \varphi_{p}(\boldsymbol{\xi})}{\partial n_{\xi}}\right) \mathrm{d} \boldsymbol{\xi} .
$$

Here we substitute the boundary conditions (4.6) and (4.8)

$$
\begin{aligned}
& \left(\begin{array}{c}
\pi \\
2 \pi
\end{array}\right) \varphi_{p}(\mathbf{x})=\int_{S_{w}} \varphi_{p}(\boldsymbol{\xi}) \frac{\partial G(\boldsymbol{\xi} ; \mathbf{x})}{\partial n_{\boldsymbol{\xi}}} \mathrm{d} \boldsymbol{\xi} \\
& +\int_{S_{g}}\left(\varphi_{p}(\boldsymbol{\xi}) \frac{\omega^{2}}{g} G(\boldsymbol{\xi} ; \mathbf{x})-G(\boldsymbol{\xi} ; \mathbf{x})\left(\frac{\omega^{2}}{g} \varphi_{p}(\mathbf{x})-\frac{i \omega}{\rho g} p\right)\right) \mathrm{d} \boldsymbol{\xi},
\end{aligned}
$$

which reduce to

$$
\left(\begin{array}{c}
\pi \\
2 \pi
\end{array}\right) \varphi_{p}(\mathbf{x})=\int_{S_{w}} \varphi_{p}(\boldsymbol{\xi}) \frac{\partial G(\boldsymbol{\xi} ; \mathbf{x})}{\partial n_{\boldsymbol{\xi}}} \mathrm{d} \boldsymbol{\xi}+\int_{S_{g}} G(\boldsymbol{\xi}, \mathbf{x}) \frac{i \omega}{\rho g} p \mathrm{~d} \boldsymbol{\xi} .
$$

We organize the equation to arrive at

$$
\int_{S_{w}} \varphi_{p}(\boldsymbol{\xi}) \frac{\partial G(\boldsymbol{\xi} ; \mathbf{x})}{\partial n_{\boldsymbol{\xi}}} \mathrm{d} \boldsymbol{\xi}-\left(\begin{array}{c}
\pi \\
2 \pi
\end{array}\right) \varphi_{p}(\mathbf{x})=-\int_{S_{g}} G(\boldsymbol{\xi}, \mathbf{x}) \frac{i \omega}{\rho g} p \mathrm{~d} \boldsymbol{\xi}
$$




\subsection{Newtonian Cooling}

\subsubsection{Applications}

\section{Numerical beach}

Newtonian Cooling is primarily used in context with Numerical beaches [6]. Numerical wave tanks are often used to analyse wave-body interaction in the time domain. To avoid reflections from the boundaries of the tank, so called numerical beaches are often applied. A numerical beach is a damping zone, where typically the wave motion is eventually damped to zero such that a homogenous Neumann boundary condition may be applied on tank boundary. In the damping zone, a dissipative term is added to the kinematic free surface boundary condition. The dissipative term, or damping term, is proportional to some damping coefficient. The damping coefficient is typically a smooth function varying with $x$. It is zero at the inner boundary of the damping zone and smoothly increases outwards until all motions are damped out.

\section{Narrow gaps and moonpools}

The Newtonian cooling method is also used as a source of external damping of fluid motion in moonpools and narrow gaps. The damping coefficient is set constant and the modified kinematic boundary condition which include the damping term is applied on the free surface in the gap, or moonpool. From a practical point of view, the method is relatively easy to implement in a potential solver. The additional damping introduced is, as we will see in our numerical study, well behaved and easy to control. However, the damping is of purely mathematical character and has no foundation in physics. The physical damping may vary greatly depending on the geometry and sea state, so the level of damping is often tuned to match those from model tests.

\subsubsection{Formulation}

The kinematic free surface condition is modified to include a damping term

$$
\frac{\partial \zeta}{\partial t}=\frac{\partial \Phi}{\partial z}-2 \nu \zeta+\frac{\nu^{2}}{g} \Phi
$$

The first additional term gives damping while the second ensures that the dispersion relation is unchanged. The additional damping is of purely mathematical character and is not related to physics. The coefficient $\nu$ will decide the level of damping and must be experimentally determined. Both the dynamic free surface boundary condition and the kinematic body boundary condition is unchanged. Combination of the kinematic and dynamic boundary condition gives the free surface condition

$$
\frac{\partial^{2} \Phi}{\partial t^{2}}+g\left(\frac{\partial \Phi}{\partial z}+\frac{2 \nu}{g} \frac{\partial \Phi}{\partial t}+\frac{\nu^{2}}{g} \Phi\right)=0 .
$$

Notice that the Green function does not satisfy this free surface condition, and a free surface integral is required, 


$$
\left(\begin{array}{c}
\pi \\
2 \pi
\end{array}\right) \varphi(\mathbf{x})=\int_{S_{w}+S_{g}}\left(\varphi(\boldsymbol{\xi}) \frac{\partial G(\boldsymbol{\xi} ; \mathbf{x})}{\partial n_{\boldsymbol{\xi}}}-G(\boldsymbol{\xi} ; \mathbf{x}) \frac{\partial \varphi(\boldsymbol{\xi})}{\partial n_{\xi}}\right) \mathrm{d} \boldsymbol{\xi} .
$$

We express the free surface boundary condition (4.14) in terms of the time independent potential

$$
\frac{\partial \varphi}{\partial z}=\frac{\omega^{2}}{g} \varphi-2 \nu \frac{i \omega}{g} \varphi-\frac{\nu^{2}}{g} \varphi,
$$

and substitute (4.16) in the integral equation (4.15),

$$
\begin{aligned}
& \int_{S_{w}} \varphi(\boldsymbol{\xi}) \frac{\partial G(\boldsymbol{\xi} ; \mathbf{x})}{\partial n_{\boldsymbol{\xi}}} \mathrm{d} \boldsymbol{\xi}+\int_{S_{g}} \varphi(\boldsymbol{\xi}) G(\boldsymbol{\xi} ; \mathbf{x})\left(\frac{2 \nu i \omega}{g}+\frac{\nu^{2}}{g}\right) d \boldsymbol{\xi}-\left(\begin{array}{c}
\pi \\
2 \pi
\end{array}\right) \varphi(\mathbf{x}) \\
& =\int_{S_{w}} G(\boldsymbol{\xi}, \mathbf{x}) \frac{\partial \varphi(\boldsymbol{\xi})}{\partial n_{\boldsymbol{\xi}}} \mathrm{d} \boldsymbol{\xi}
\end{aligned}
$$

We note that $\varphi$ is an unknown on the wetted boundary and the gap surface at the same time regardless of the location of $\mathbf{x}$. 


\section{Chapter 5}

\section{Numerical method and program structure}

\subsection{Boundary element method}

We distinguish between two different methods frequently used within BEM to solve potential theory problems. Those are the potential formulation and the source formulation. The potential formulation is often the method of choice when the free surface Green function is known, and its derivatives and integrals are easily evaluated. As noted in 2.2, the proper choice of Green function traditionally reduce the integrals to be taken only over the body surface, as the free surface integral is zero. In the source formulation one use the simple Rankine source and seek to determine its strength. However, this requires meshing of the free surface. In the present study we are applying the potential formulation to solve the Laplace equation together with the given boundary conditions. The potential formulation utilize the fact that Greens theorem may be used to formulate integral equations for the unknown velocity potential. We have already developed the integral equations for our desired potential solutions in Chapter 4 , as well as for the basis problem in 2.2. These equations are discretisized to form a linear set of algebraic equations. Typically, the body surface is divided into $N$ segments, or panels. A panel distribution is illustrated in figure 5.1. Over each of these panels the velocity potential is assumed constant. The point where the potential is to be evaluated is referred to as the field point $\mathbf{x}_{i}$, while singularities are distributed at the source points $\boldsymbol{\xi}_{j}$. The singularities are the pulsating source and dipole, given as the Green function (2.19) and its normal derivative. The numerical integrals over each panel are often carried out with Gaussian quadrature. This technique yields better convergence than for example the trapezoidal rule. In general more panels are required for the latter method. However, in our implementation we are using the trapezoidal rule for simplicity. Convergence is demonstrated in Appendix C.

\subsubsection{System without additional damping}

First, the general procedure are presented for the traditional integral equation (2.21) without additional free surface terms. We consider the field point $\mathbf{x}_{i}$ on 

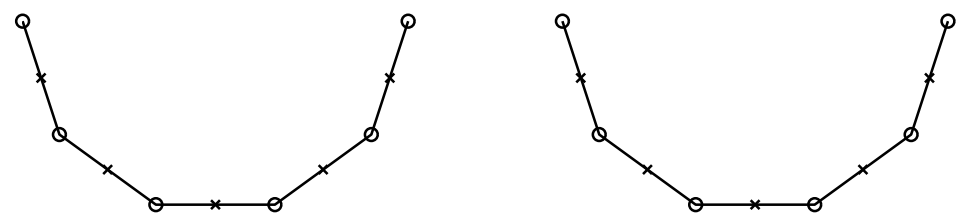

Figure 5.1: Example of a panel discretization of a double-hull consisting of two half circles. Panel vertices and centroids are marked by circles and cross respictively.

the body boundary. The discretized integral equation for the potential at field point $\mathbf{x}_{i}$ takes the form

$$
\sum_{j=1}^{N} \varphi_{j} \int_{S_{w_{j}}} \frac{\partial G_{i j}}{\partial n_{j}} \mathrm{~d} \xi_{j}-\pi \varphi_{j} \delta_{i j}=\sum_{j=1}^{N} \frac{\partial \varphi_{j}}{\partial n_{j}} \int_{S_{w_{j}}} G_{i j} \mathrm{~d} \xi_{j}
$$

Notice that both $\varphi_{j}$ and $\frac{\partial \varphi_{j}}{\partial n_{j}}$ are moved outside of the integration as they are assumed constant over each panel. We introduce the term influence coefficient matrix and denote it by $A_{i j}$,

$$
A_{i j}=\int_{S_{w_{j}}} \frac{\partial G_{i j}}{\partial n_{j}} \mathrm{~d} \xi_{j}-\pi \delta_{i j}
$$

where $\delta_{i j}$ is the Kronecker-delta, equal to 1 if $i=j$ and zero otherwise. The influence coefficient matrix represents the normal velocity induced on the $j$ 'th panel by a unit-density source distribution on the $i$ 'th panel. We denote the right hand side column vector as $b_{i}$,

$$
b_{i}=\sum_{j=1}^{N} \frac{\partial \varphi_{j}}{\partial n_{j}} \int_{S_{w_{j}}} G_{i j} \mathrm{~d} \xi_{j}
$$

Now we can write the set of linear equations as

$$
\sum_{j=1}^{N} A_{i j} \varphi_{j}=b_{i}
$$


This matrix equation is solved by Gaussian elemination.

When the potential on the body is known, (5.1) can be used to find the potential at field point $\mathbf{x}_{l}$ located anywhere in the fluid.

$$
\varphi_{l}=\frac{1}{2 \pi} \sum_{j=1}^{N} \int_{S_{w_{j}}}\left(\varphi_{j} \frac{\partial G_{l j}}{\partial n_{j}}-G_{l j} \frac{\partial \varphi_{j}}{\partial n_{j}}\right) \mathrm{d} \xi_{j} .
$$

The potential solution in the gap is necessary to compute the gap surface elevation. To get a continous representation of the solution we discretize the gap surface $S_{g}$ into $M$ panels, and solve (5.5) for each $l$ on $S_{g}$.

\subsubsection{System with free surface damping}

In the integral equations for the free surface damping methods, a non-zero free surface integral is present in the gap. This requires paneling of the gap surface and additional gap surface integrals are introduced. We denote field points on $S_{g}$ by $l$ and the source points by $k$.

\section{Integral equations for the Pressure Damping Model}

The total pressure damped solution is expressed as a superposition of the radiation potential, the diffraction potential and the pressure generated potential. The radiation and diffraction potential is the same as in the system without additional damping and the solution procedure is discribed by 5.1.1. The integral equation for the pressure generated potential is given in (4.12). We discretize it in a similar manner as (5.1). For $\mathbf{x}$ on $S_{w}$ we get

$$
\sum_{j=1}^{N} \varphi_{j}^{p} \int_{S_{W_{j}}} \frac{\partial G_{i j}}{\partial n_{j}} \mathrm{~d} \xi_{j}-\pi \varphi_{j}^{p} \delta_{i j}=-\sum_{k=1}^{M} \frac{i \omega}{\rho g} p \int_{S_{g_{k}}} G_{i k} \mathrm{~d} \xi_{k},
$$

while the equation for $\mathbf{x}$ on $S_{g}$ is given by

$$
\sum_{j=1}^{N} \varphi_{j}^{p} \int_{S_{W_{j}}} \frac{\partial G_{l j}}{\partial n_{j}} \mathrm{~d} \xi_{j}-2 \pi \varphi_{j}^{p} \delta_{l k}=-\sum_{k=1}^{M} \frac{i \omega}{\rho g} p \int_{S_{g_{k}}} G_{l k} \mathrm{~d} \xi_{k} .
$$

The left hand side matrix in (5.6) is identical to that in (5.1), while the right hand side is the contribution from the non-zero gap surface integral. When $p$ is a function of the velocity obtained from the basis problem, as in (4.5), it is required that(5.1) and (5.5) is solved at first.

\section{Integral equations for the Newtonian cooling model}

The additional Newtonian cooling terms affect the left hand side of the integral equation (5.8), as the new terms contains the unknown velocity potential. For $\mathrm{x}$ on $S_{w}$ the discretisized equation reads 


$$
\begin{aligned}
& \sum_{j=1}^{N}\left(\varphi_{j} \int_{S_{w_{j}}} \frac{\partial G_{i j}}{\partial n_{j}} \mathrm{~d} \xi_{j}-\pi \varphi_{j} \delta_{i j}\right)+\sum_{k=1}^{M} \varphi_{k} \int_{S_{g_{k}}} G_{i k}\left(\frac{2 \nu i \omega}{g}+\frac{\nu^{2}}{g}\right) d \xi_{k} \\
& =\sum_{j=1}^{N} \frac{\partial \varphi_{j}}{\partial n_{j}} \int_{S_{w_{j}}} G_{i j} \mathrm{~d} \xi_{j}
\end{aligned}
$$

For $\mathbf{x}$ on $S_{g}$ we get the following

$$
\begin{aligned}
& \sum_{j=1}^{N} \varphi_{j} \int_{S_{w_{j}}} \frac{\partial G_{l j}}{\partial n_{j}} \mathrm{~d} \xi_{j}+\sum_{k=1}^{M}\left(\varphi_{k} \int_{S_{g_{k}}} G_{l k}\left(\frac{2 \nu i \omega}{g}+\frac{\nu^{2}}{g}\right) d \xi_{k}-2 \pi \varphi_{j} \delta_{l k}\right) \\
& =\sum_{j=1}^{N} \frac{\partial \varphi_{j}}{\partial n_{j}} \int_{S_{w_{j}}} G_{l j} \mathrm{~d} \xi_{j}
\end{aligned}
$$

The two equations, 5.8 and 5.9, must be solved simultaneously due to that the velocity potential is unknown on both $S_{w}$ and $S_{g}$ regardless of the location of $\mathbf{x}$. The matrix equation $\mathbf{A} \varphi=\mathbf{b}$ is arranged as

$$
\left(\begin{array}{ll}
A & B \\
C & D
\end{array}\right)\left(\begin{array}{l}
\varphi_{1} \\
\varphi_{2}
\end{array}\right)=\left(\begin{array}{l}
b_{1} \\
b_{2}
\end{array}\right)
$$

where elements of $A$ and $b_{1}$ is given in 5.2 and 5.3, while the rest of the vectors and matrices are given as

$$
\begin{aligned}
& B_{i k}=\int_{S_{g_{k}}} G_{i k}\left(\frac{2 \nu}{g} i \omega+\frac{\nu^{2}}{g}\right) \mathrm{d} \xi_{k} \\
& C_{l j}=\int_{S_{w_{j}}} \frac{\partial G_{l j}}{\partial n_{j}} \mathrm{~d} \xi_{j} \\
& D_{l k}=-2 \pi \delta_{l k}+\int_{S_{g_{k}}} G_{l k}\left(\frac{2 \nu}{g} i \omega+\frac{\nu^{2}}{g}\right) \mathrm{d} \xi_{k} \\
& b_{2, l}=\sum_{j=1}^{N} \frac{\partial \varphi_{j}}{\partial n_{j}} \int_{S_{w_{j}}} G_{l j} \mathrm{~d} \xi_{j}, \\
& \varphi_{1, j}=\varphi_{j}, \\
& \varphi_{2, k}=\varphi_{k},
\end{aligned}
$$

for $i, j=1, \ldots, N$ and $l, k=1, \ldots, M$. 


\subsection{Program structure}

It is appropriate to demonstrate how both the additional damping methods may be included in a traditional BEM potential solver. Our approach is based on that equation (5.1) and (5.5) are solved at first by our basis solver. As noted in 5.1.2, the influence coefficient matrices and right hand side vectors in the Pressure Damping model and the Newtonian cooling model, are equal or partially equal those in the basis problem. This enables opportunities for effective free surface damping solvers as add-ons to the basis solver. The layout for the BEM-code developed for the present study is outlined in figure 5.2.

Note: Parts of the basis solver were developed by the author in an earlier project work, while the rest of the program were developed during the work with this thesis. 


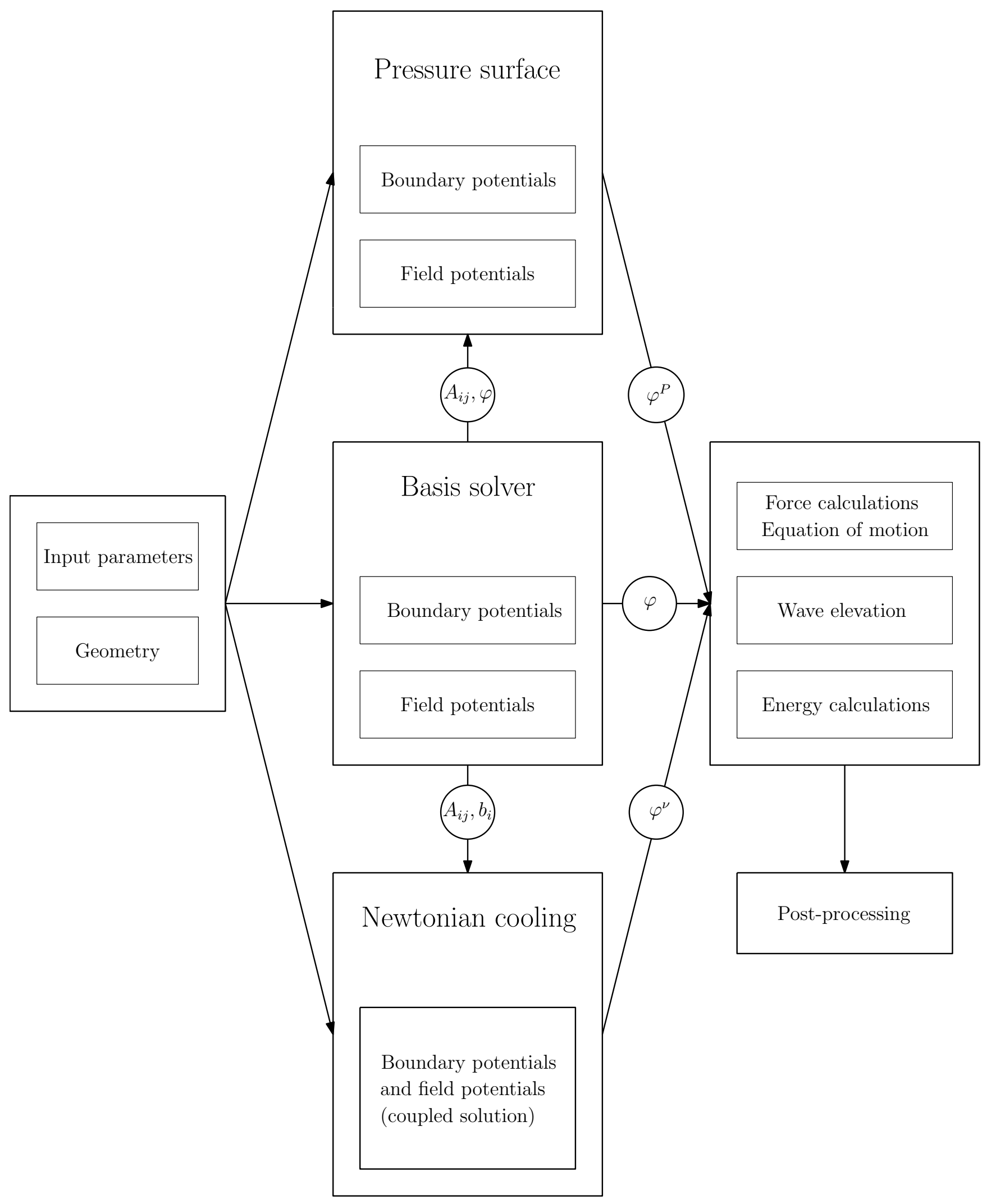

Figure 5.2: Program structure of the BEM code developed for this thesis. 


\section{Chapter 6}

\section{Numerical Analysis and Results}

\subsection{Introductory comment}

The author is aware of that the computed gap wave elevation in the basis problem is not completely consistent with those presented by Kristiansen and Faltinsen [8]. Their reported amplitude at resonance in forced heave motion is $A_{g} /\left|\xi_{3}\right| \approx 13$, while our calculations produce an amplitude $A_{g} /\left|\xi_{3}\right| \approx 16$. Despite this, the gap resonance frequency and the far-field amplitude shows good correspondence. The cause of the disagreement for the gap resonance amplitude has not been found. Different results from our code is controlled by energy considerations and far-field relations in the following sections, and convergence is demonstrated in Appendix C.

\subsection{The basis problem}

We will first present results from our analysis of wave-body interaction when no additional damping is applied in the gap. Although the calculations without any additional damping overpredict the gap elevation, it will provide important understanding of the dynamics and the interaction between different parts of the system. We do a complete analysis of a double-hull, including the radiation and diffraction subproblems, and the situation when the body is freely floating. We seek to illustrate the previously described gap resonance, body resonance and their interaction. Energy and farfield relations will serve as code validation. Some of our results will also be compared to output from the three-dimensional potential solver Wasim provided by DNV GL Software. In this context threedimensional effects will be briefly discussed.

\subsubsection{Forced heave motion}

In the radiation problem we solve the velocity potentials due to forced body motion in the relevant degrees of freedom. In the two-dimensional case we have three degrees of freedom, namely heave, sway and roll. Only heave motion will contribute to the piston mode elevation, as sway and roll motion will result in 
anti-symmetric wave modes. The resonance frequencies for the sway and heave induced wave occurs at higher frequencies than the piston mode resonance. The anti-symmetric effects are nearly zero around the piston mode resonance. The piston mode behaviour is found by investigating forced heave motion in the frequency range where the piston mode frequency occurs. Higher order gap resonance frequencies where antisymmetric modes are trigged may be found by studying sway and heave motion for higher frequency ranges. Higher order symmetric modes from heave motion may be found as well. In our study we focus our attention on the piston mode behaviour, and results from the forced heave motion analysis are discussed. Of main interest is the gap surface elevation amplitude and the force acting on the hull. Figure 6.1 illustrates forced heave motion of the body.

The gap surface elevation is computed for $x$ in the center of the gap. We have also computed the wave amplitude in the far-field region with $x=20 B$ away from the body. The amplitudes are displayed in figure 6.2 , where $A_{g 0}$ is in the gap while $A_{f 0}$ is in the far-field. Large amplitudes occurs near $\omega^{2} B / g \approx$ 1.05. This is the gap resonance frequency. The location of this frequency is independent on the body mass properties, thus it is only dependent on the geometric values. Around this frequency the wave oscillation will change rapidly from being in phase with the heave motion, to beeing $180^{\circ}$ out of phase with the heave motion. The behaviour of the far-field wave amplitude is closely related to the wave amplitude in the gap. The radiated waves is not only affected by the forced body motion, but is also greatly influenced by the response in the gap. A noteworthy phenomenon occurs around $\omega^{2} B / g \approx 1.2$, where the radiated wave amplitude is zero. Here the coupled effect of the forced body motion and the response in the gap will cancel out and no outgoing wave is generateted.

The pressure on the body surface is found from Bernoulli's equation (2.5) by substituting (2.12), and the pressure force is found by integrating the pressure over the body surface,

$$
F_{i}=\operatorname{Re}\left\{i \omega \xi_{j} e^{i \omega t} f_{i j}\right\}
$$

where

$$
f_{i j}=-\rho i \omega \int_{S_{b}} n_{i} \varphi_{j} \mathrm{~d} S .
$$

The hydrodynamic coefficients, added mass and damping, is then defined as

$$
\begin{aligned}
& a_{i j}=\operatorname{Re}\left\{f_{i j} / \omega^{2}\right\}, \\
& b_{i j}=-\operatorname{Im}\left\{f_{i j} / \omega\right\} .
\end{aligned}
$$

Due to symmetri, only $f_{33}$ contributes to the integral (6.2) in heave motion. Dimensionless added mass and damping coefficient is displayed in figure 6.3. 


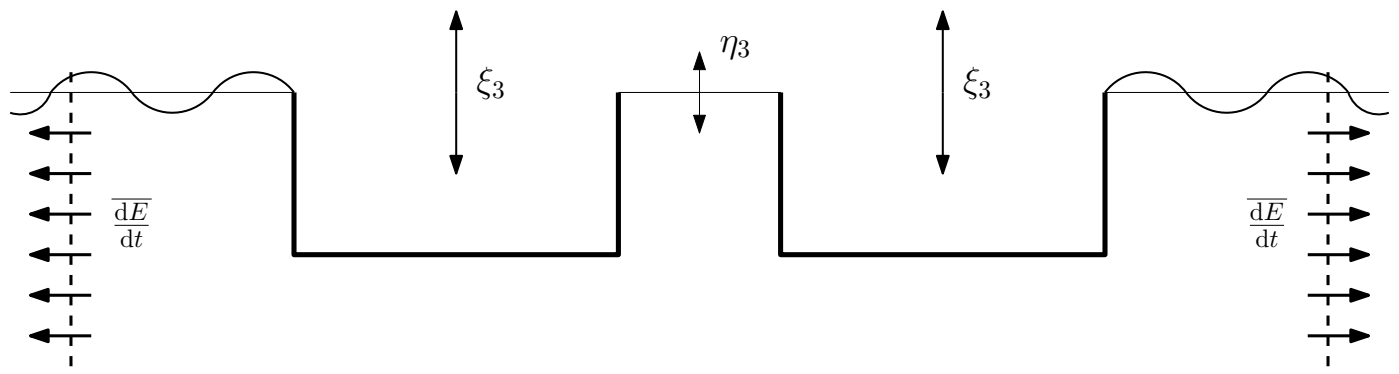

Figure 6.1: Forced heave motion with forcing amplitude $\left|\xi_{3}\right|$. The forced body motion generates oscillation in the gap, and radiated waves with mean energy flux $\frac{\overline{\mathrm{d} E}}{\mathrm{~d} t}$. Our main interest is the wave amplitude $\left|\eta_{3}\right|$ in the gap.

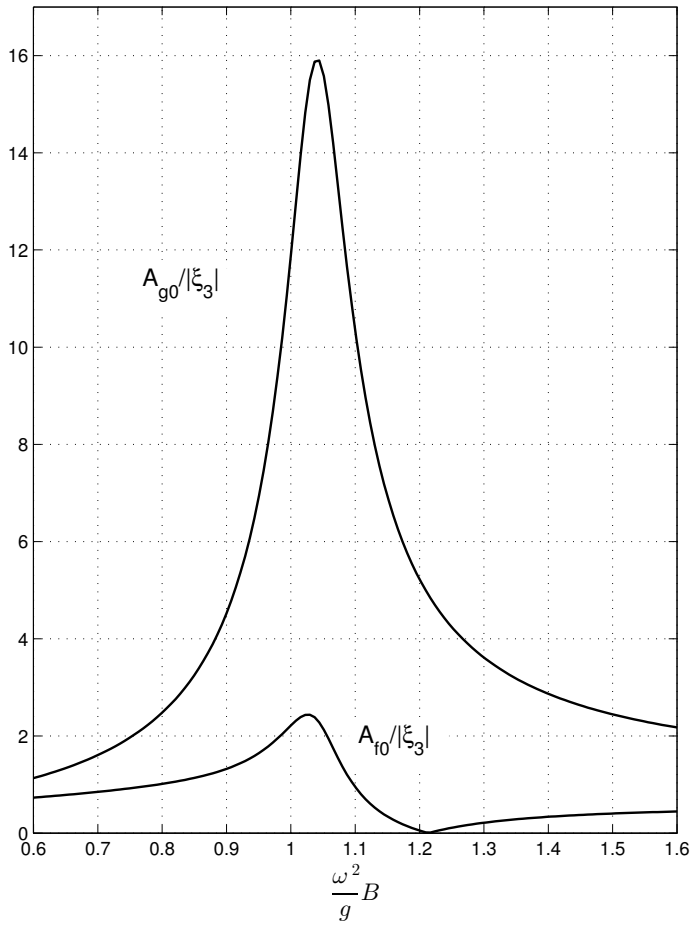

Figure 6.2: Wave amplitude $A_{g 0}=\left|\eta_{3}\right|$ in the gap, and $A_{f 0}=\left|\eta_{3}\right|$ in the far field, relative to the forcing amplitude $\left|\xi_{3}\right| . B / D=2, b / D=1$.

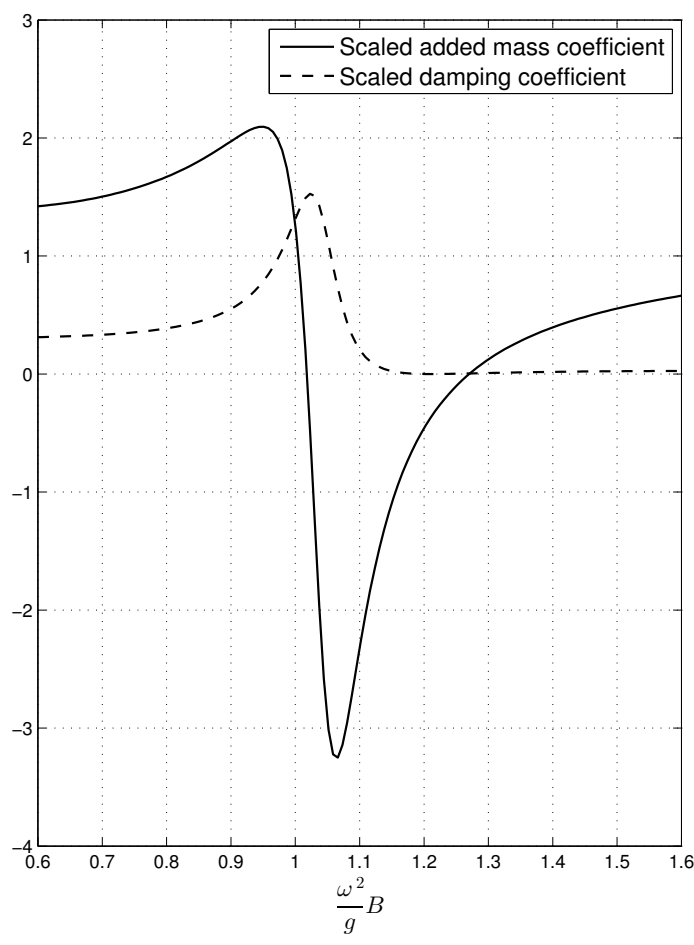

Figure 6.3: Hydrodynamic coefficients. Scaled added mass coefficient $a_{33} / \rho B^{2}$, and scaled damping coefficient $b_{33} /\left(\rho B^{2} \sqrt{\rho B}\right)$. 
The damping coefficient is directly related to the energy of the outgoing waves. The average work done by the body on the fluid over one period is given as

$$
\bar{W}=-\overline{F_{3} U_{3}}=\frac{1}{2} \omega^{2} b_{33}|\xi|^{2}
$$

The energy added to the waves must radiate outwards and may alternatively be found by computing the energy flux in the far field. For infinite depth, the average energy flux is

$$
\frac{\overline{d E}}{d t}=V_{g} \bar{E}
$$

where $V_{g}$ is the group velocity and the energy density $\bar{E}$ is given by

$$
\bar{E}=\frac{1}{2} \rho g A_{f}^{2},
$$

where $A_{f}$ is the far-field amplitude. In this context, this relation will serve as an excellent code check. Computed values of (6.12) and (6.5) is shown in figure 6.4 .

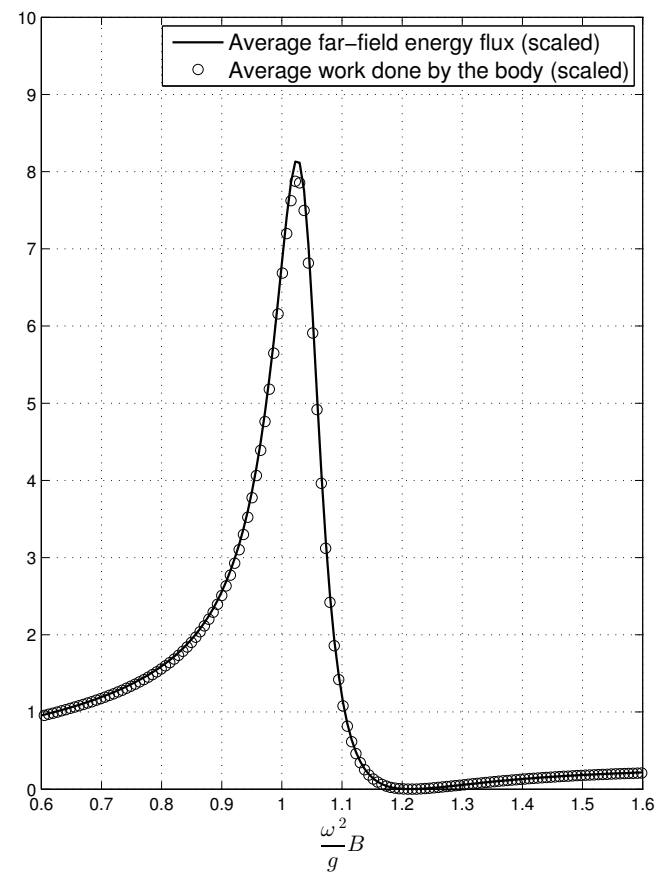

Figure 6.4: Average work done over one period scaled as $\bar{W} /(\rho B g \sqrt{B g})$, and average energyflux over one period scaled as $\frac{\overline{d E}}{d t} /(\rho B g \sqrt{B g})$. 


\subsubsection{Incident wave upon rigid double-hull}

We let an incident wave interact with the body when it is restrained from moving. This is the diffraction problem, illustrated in figure 6.5. The incident wave will induce wave motion in the gap. The computed amplitude is shown in figure 6.6. The peak amplitude occur at the gap resonance frequency.

The pressure is found from Bernoulli's equation (2.5) by substituting (2.14), and the excitation force is found by integrating the pressure over the body surface,

$$
F_{i}=\operatorname{Re}\left\{A X_{i} e^{i \omega t}\right\}
$$

where the complex amplitude $X_{i}$ is defined as

$$
X_{i}=-\rho \int_{S_{w}}\left(\varphi_{0}+\varphi_{s}\right) \mathrm{d} S
$$

An alternative formulation for the excitation force is given from the Haskind relation (e.g. see Newman 1977, ch. 6.18) as

$$
\left|X_{i}\right|^{2}=2 \rho g V_{g} b_{i i}
$$

In amplitude of the exciting force is displayed in figure 6.7 , where it has been computed using 6.8 and 6.9 . 


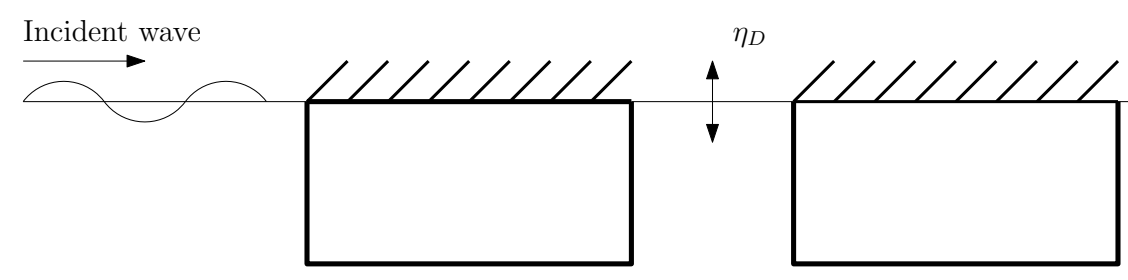

Figure 6.5: Incident wave interacting with fixed bodies.

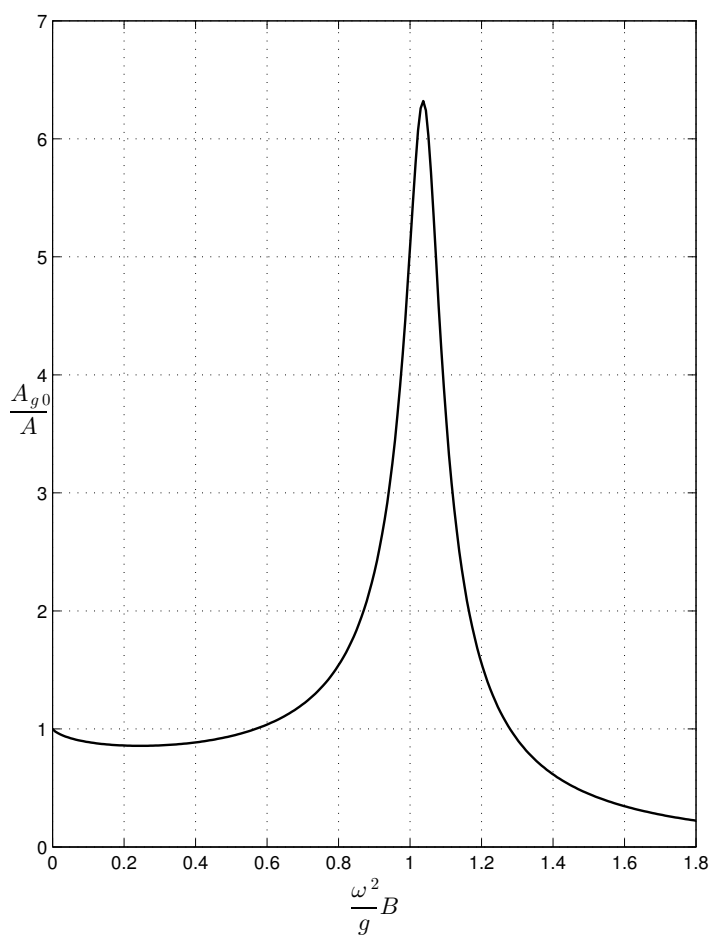

Figure 6.6: Wave amplitude $A_{g 0}=\left|\eta_{D}\right|$ in the gap, relative to the incident wave amplitude $A . B / D=2$, $b / D=1$.

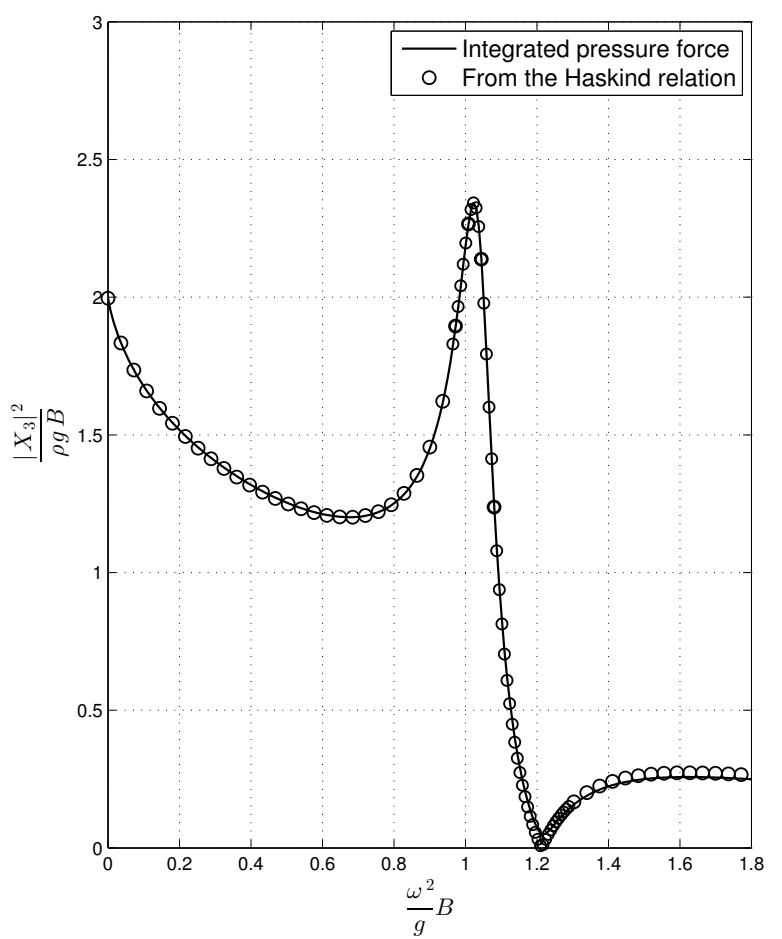

Figure 6.7: Scaled excitation force amplitude computed from direct pressure integration and the Haskind relation. 


\subsubsection{Incident wave upon freely floating body}

When the body is free to respond to an incident wave, the fluid motion is dependent on both the diffraction, and the radiation induced by the body response. The body response will again be dependent on the surrounding fluid motion. The presence of a gap or moonpool will greatly influence the situation.

The body motion is a result of the forces acting on the body. As presented in 6.2.1 and 6.2.2, the radiation forces are proportional to the body motion itself, while the excitation force is proportional to the amplitude of the incident wave. In addition to these forces we have the inertia force and the restoring force. The equation of motion is then given by

$$
\xi_{3}\left(-\omega^{2}\left(M_{33}+a_{33}\right)+i \omega b_{33}+c_{33}\right)=A X_{3},
$$

where $c_{33}$ is the heave restoring force and $M_{33}$ is the mass of the body. Details on 6.10, the restoring force matrix $c_{i j}$ and mass matrix $M_{i j}$ is given in Appendix D. Equation (6.10) is solved for the unknown response amplitude $\xi_{3}$. By dividing both sides by $A$, we get the Response Amplitude Operator (RAO), which describes the ratio between the body response amplitude and the amplitude of the incident wave.

The piston mode elevation in the gap, relative to the incident wave amplitude, is displayed in figure 6.9, with the corresponding RAO in figure 6.10. A particularly interesting phenomenon is the cancellation of large gap surface elevation occuring at the gap resonance frequency in the radiation and diffraction problem. The reason for this is that the diffracted wave component is of approximately equal amplitude as the radiation wave component, but of opposite phase at this frequency. Instead, the peak wave elevation coincides with the peak body response. The phase angle of the gap elevation and the body motion is shown in figure 6.11. Three important frequencies has been marked by lines labeled as 1, 2 and 3. Line 1 represents the gap resonance frequency, where no special incidents occur due to cancellation of the radiated wave and the scattered wave. The line marked by 2 represents the frequency where the damping coefficient, as well as the excitation force, is zero in figure 6.3 and 6.7. We observe that the phase shifts by $\pi$ radians in this area. Line 3 represents the frequency where peak body motion and peak elevation occurs. Both the elevation and body motion experience a phase shift of $\pi$ radians around this frequency.

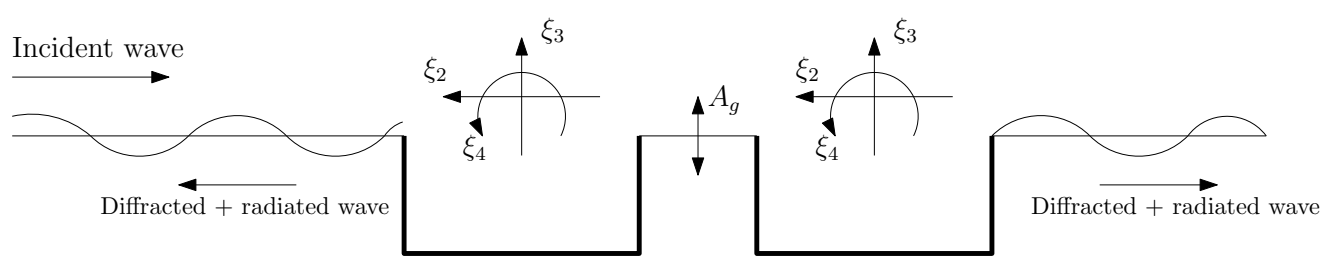

Figure 6.8: Freely floating body. Different wave contributions are illustrated with arrows. 


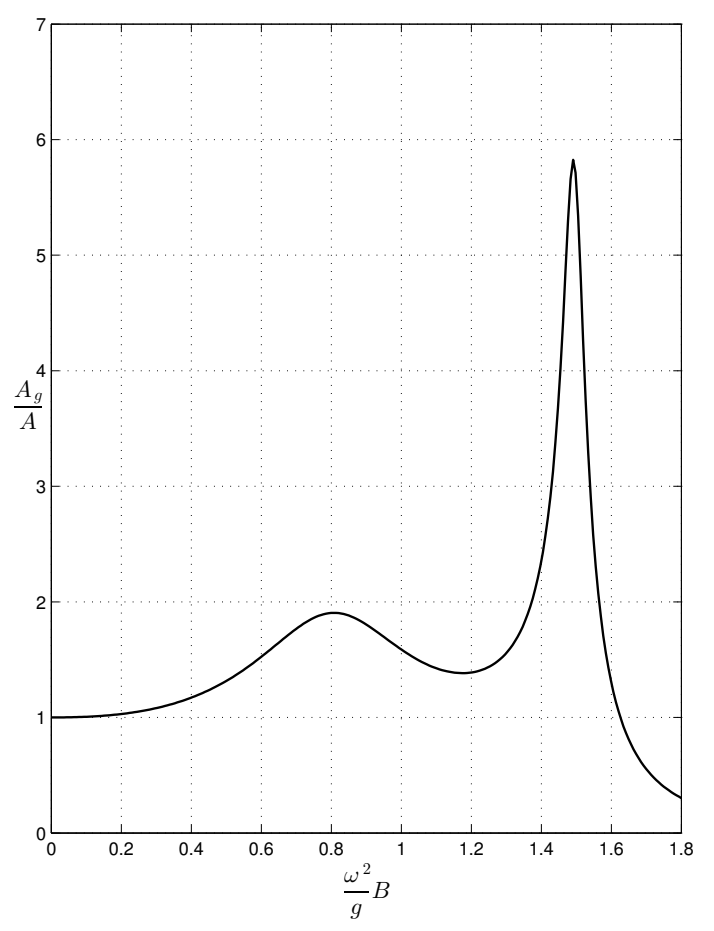

Figure 6.9: Wave elevation in gap. $B / D=2, b / D=1$.

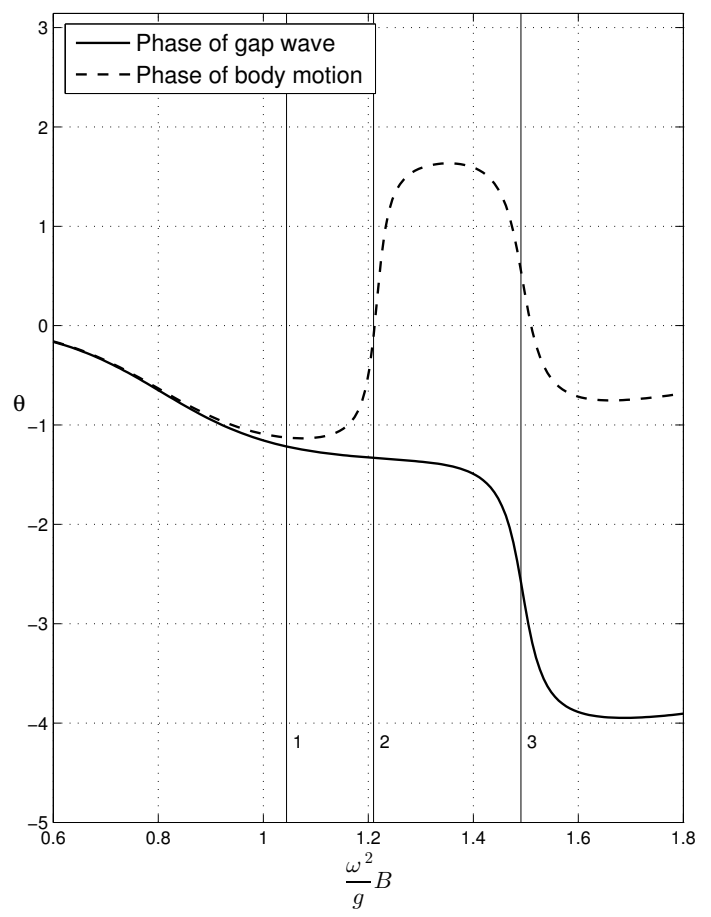

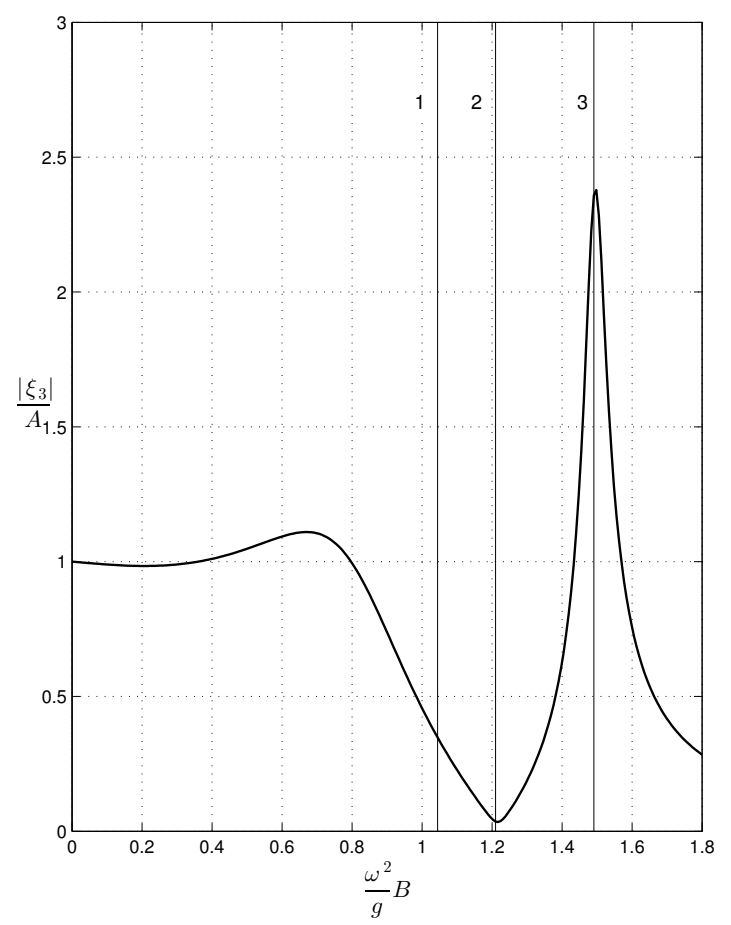

Figure 6.10: Heave response amplitude operator. The lines marked by 1,2 and 3 indicates the gap resonance frequency, the frequency where the damping is zero, and the peak body responce frequency respectively. $B / D=2, b / D=1$.
Figure 6.11: Phase of the body motion and the pistonlike elevation in the gap. The lines marked by 1,2 and 3 indicates the gap resonance frequency, the frequency where the damping is zero, and the peak body responce frequency respectively. $B / D=2, b / D=1$. 


\subsubsection{Comparison with $3 \mathrm{D}$ results from Wasim and com- ments on three dimensional effects}

By comparing results from the two dimensional (2D) case to those from a three dimensional (3D) analysis, insigths to three dimensional effects is obtained. The $3 \mathrm{D}$ computations are performed with the time domain potential solver Wasim from the HydroD package provided by DNV GL Software. The investigated case is similar to the $2 \mathrm{D}$ analysis presented in 6.2 .3 , but with circular cylinders. A $2 \mathrm{D}$ analysis with equivalent cross section were done to get comparable results. The three dimensional floating body consists of two rigidly connected half circular cylinders with length $L=10 \mathrm{~m}$, radius $R=1 \mathrm{~m}$ and with a gap width $b=0.8 \mathrm{~m}$. The geometry and the panel mesh is displayed in figure $6.12,6.13$ and 6.14 .

The body is free to respond to beam sea in heave while the other degrees of freedom are restrained. Obtained heave response is still the same as the one obtained if the body were freely floating. More specifically, pitch and surge is zero due to symmertry, while there is no coupling between heave and sway, or heave and roll.

The wave amplitude at the center of the gap is displayed together with the result from $2 \mathrm{D}$ computations in figure 6.17. The heave response is shown in figure 6.16. Both the 3D computed wave amplitude and heave RAO shows good resemblance to the ones from the $2 \mathrm{D}$ case, but with some evident differences. Differences is to be expected as the three dimensional case allows for variation of the elevation in the gap length direction. In the region $\omega^{2} R / g \approx 1.5$ to $\omega^{2} R / g=2.5$ the wave amplitude in 3D shows local maximums with fairly large amplitude whereas the 2D amplitude is relatively small. A striking difference in the compared heave responses occurs where the $2 \mathrm{D}$ response has its peak value. A possible explanation to this might be that a part of the energy in the incident wave radiate outward with waves in the gaps longitudinal direction rather than doing work on the body. The peak wave amplitude in $2 \mathrm{D}$ is directly related to the body response. This is not that apparent in $3 \mathrm{D}$, as the peak wave amplitude occurs even though the body response has a local minimum. The elevation in the gap is presumably greatly affected by allowing outgoing waves in the lingitudinal direction. This suggests that the noted local maximums in the wave amplitude is related to these outgoing waves. It is also presumable that the good agreement between 2D and 3D wave amplitude at the global maximum is somewhat random. Presence of waves in the longitudinal direction are demonstrated in figure 6.17. 


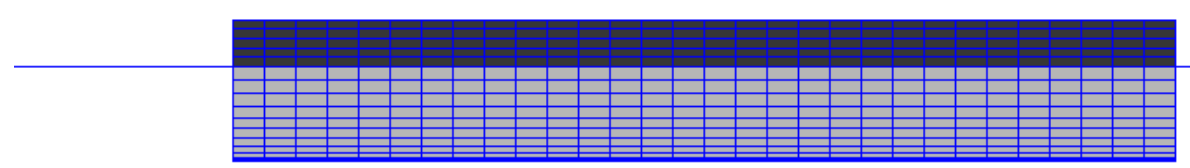

Figure 6.12: Side view of the body mesh. The length of the body is $L=10 \mathrm{~m}$. The darker area of the mesh is above the free surface.

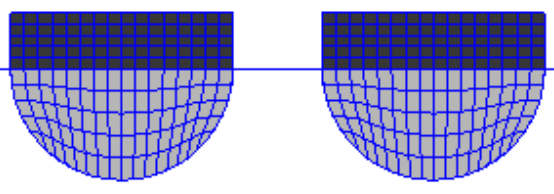

Figure 6.13: Front view of the body mesh. The radius of the submerged cylinder is $R=1.0 \mathrm{~m}$, while the gap width is $b=0.8 \mathrm{~m}$. The darker area of the mesh is above the free surface.

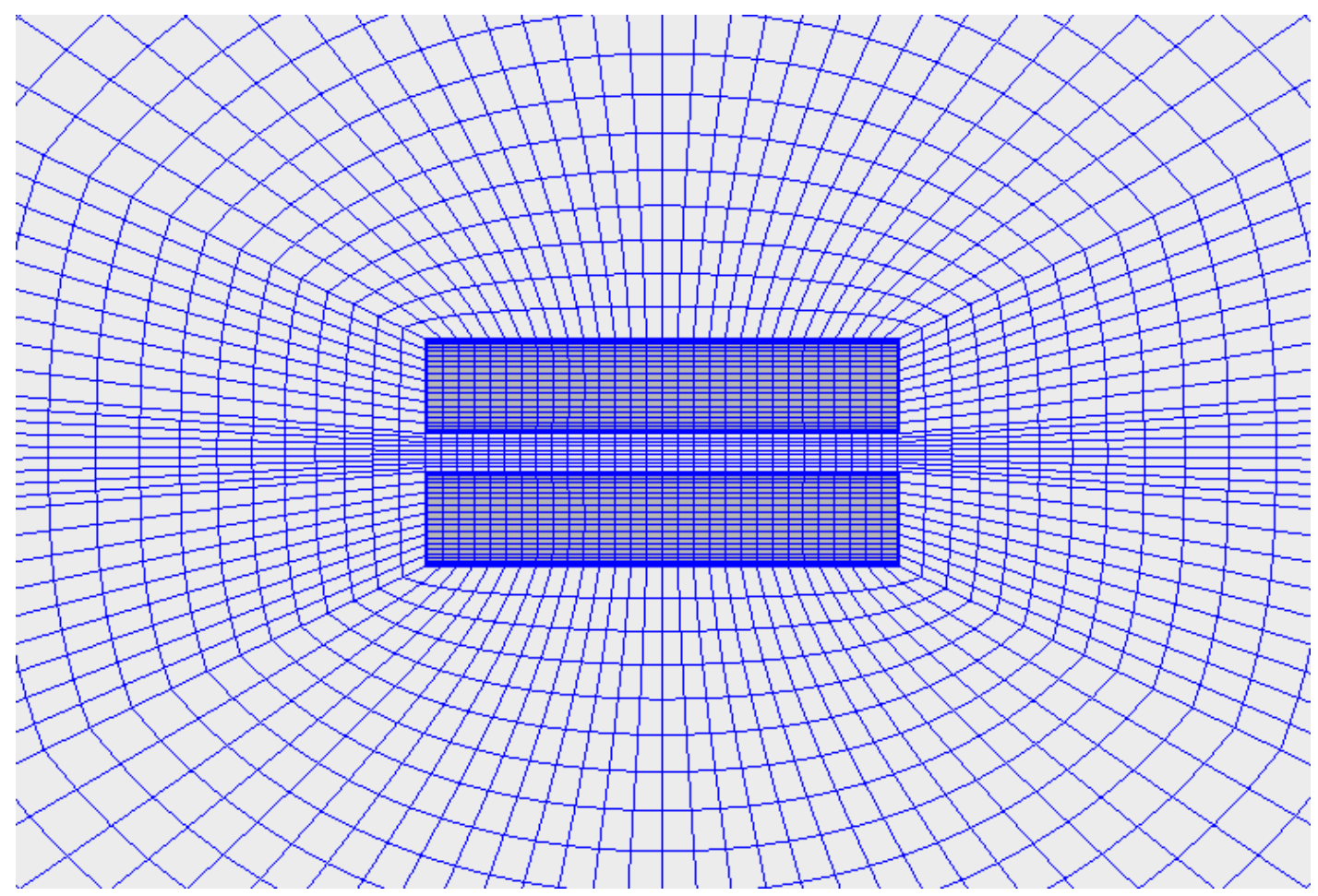

Figure 6.14: Top view showing the body and a portion of the free surface mesh around the body. 


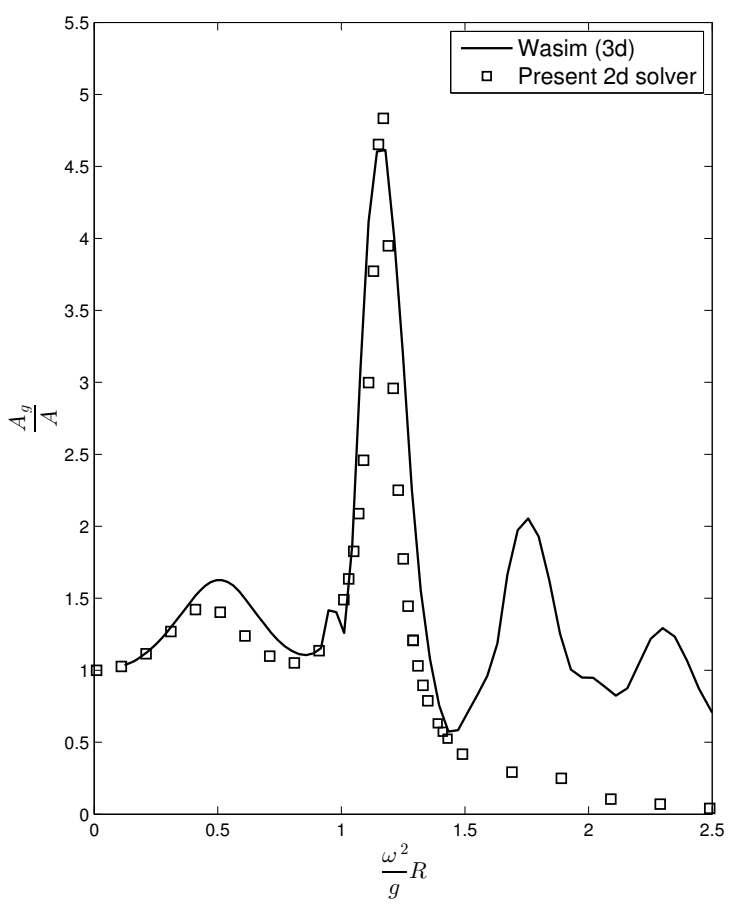

Figure 6.15: Dimensionless wave amplitude at the center point of the gap.

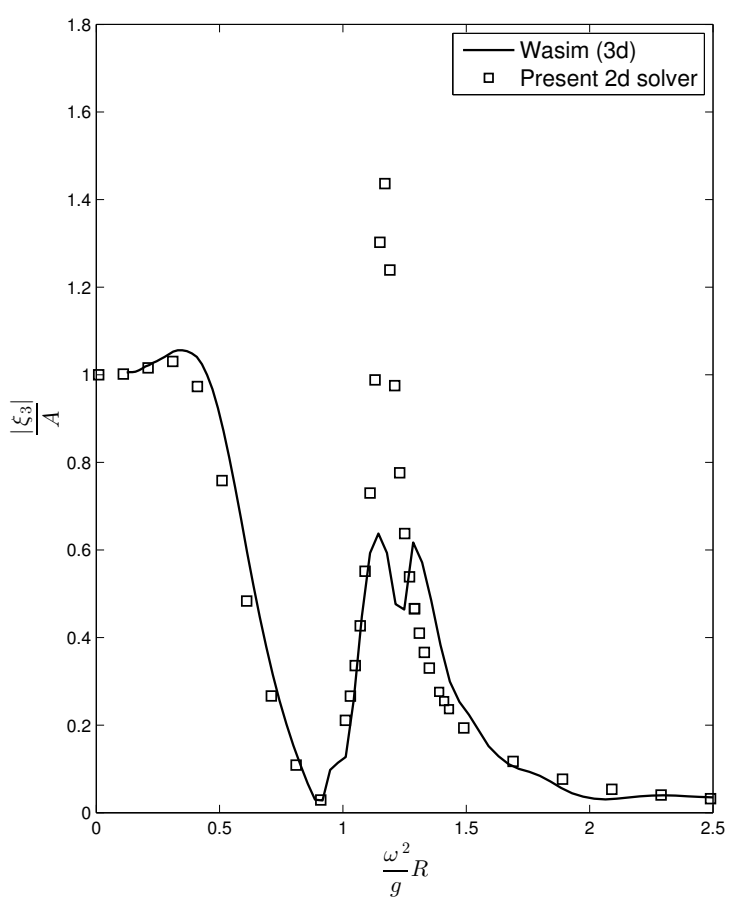

Figure 6.16: Heave RAO for the pair of circular cylinder in beam sea.

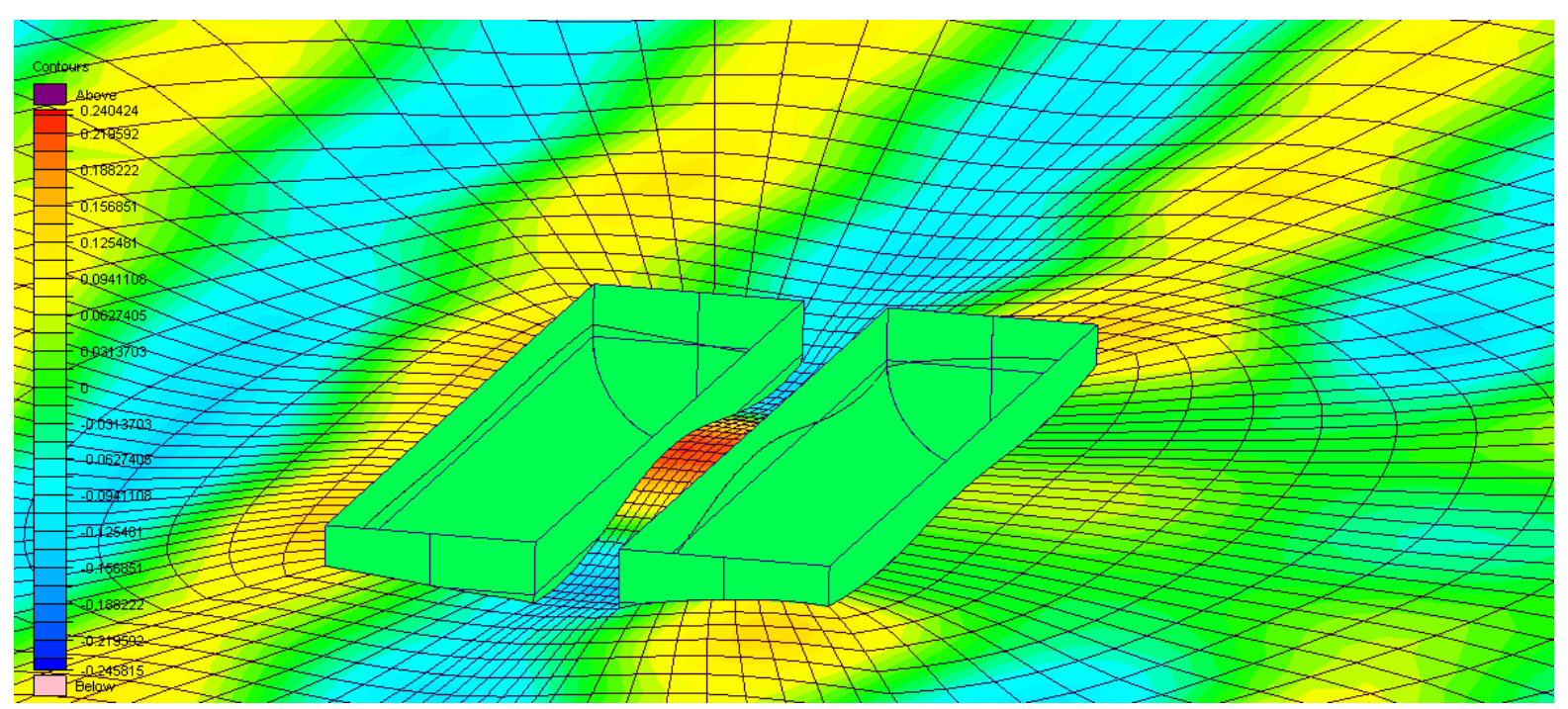

Figure 6.17: Beam sea with amplitude 0.1m upon rigidly connected cylinders for a given wave frequency. The figure illustrates variations along the gap length which is not present in the two-dimensional case. 


\subsection{Free surface pressure model}

\subsubsection{Introductory remarks}

The wave contribution caused by the free surface pressure will be referred to as the pressure generated wave in this discussion. The viscous force defined in 3.5 will always act in the opposite direction of the fluid velocity in the gap. When the force is represented by a free surface pressure, an equivalent requirement is that the pressure generated wave contribution must be in opposoite phase of the undamped wave. This will not happen if the pressure is given the opposite phase of the fluid velocity. The pressure generated wave will undergo a phase shift at resonance, just as the undamped wave, so if the pressure is given a phase shift at resonace, the pressure generated wave will experience a double phase shift. From the following study of forced heave motion we found that the pressure should have the same phase as the body motion to behave correctly at resonance. Figure 6.18 shows the phase angles for the wave trigged by forced body motion, the wave generated by the free surface pressure and the total wave consisting og both contributions. The total wave will have the same phase as the motion generated wave contribution as long as the amplitude of the pressure generated wave is less than the amplitude of the motion generated wave.

An other issue is related to the fact that the pressure is non-linear in the amplitude of the undamped solution in our formulation. In heave motion this may result in the behaviour shown in figure 6.19 when the forced motion amplitude exceeds a certain value. It is our belief that this is unphysical, and that it occur because we use the undamped amplitude in the formulation for the pressure.

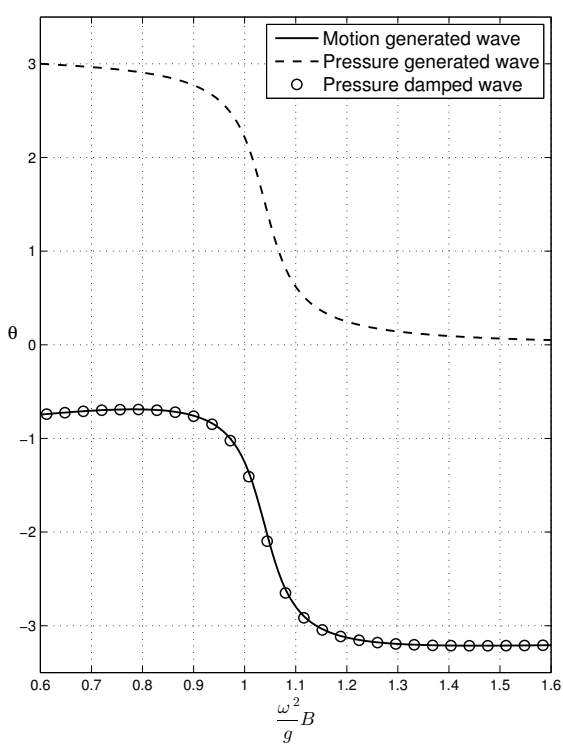

Figure 6.18: Phase angles for the wave induced by forced body motion, the pressure generated wave and the total sum.

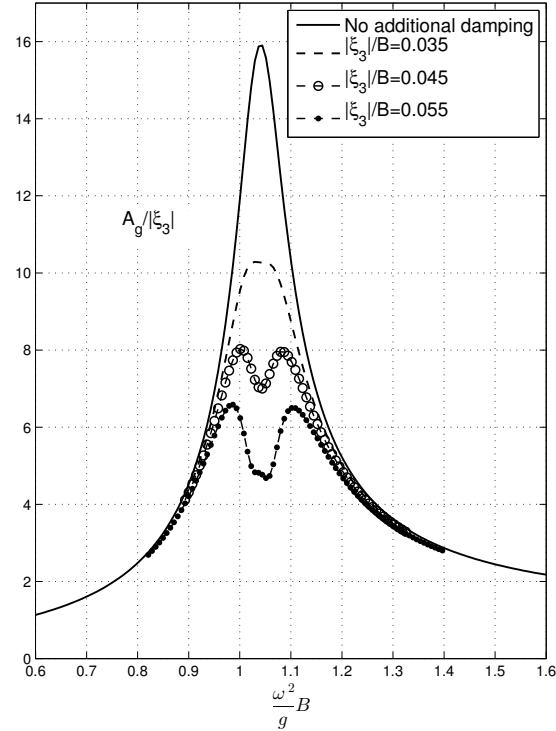

Figure 6.19: Local minimum at resonance may occurs due to that the pressure is non-linear in the amplitude of the undamped solution. 


\subsubsection{Heaving of rectangular boxes}

As an application of the Pressure Damping Method, we will perform an in-depth analysis of two closely spaced boxes in forced harmonic heave motion with an oscillating pressure distribution on the gap surface. The pressure distribution is related to the viscous forces as in section 4.2 . We investigate the local effects in means of wave amplitude and phase, force coefficients and gap energy density. The effect in the far-field is investigated through far-field amplitudes and energy flux.

Due to the formulation of the pressure amplitude, the pressure damped wave will be dependent on the amplitude of the heave oscillation. Different heave amplitudes are considered.

We have discussed different sources of viscous damping; skin friction, vortex shedding from corners and bilge keel effects. The analysis will indicate the relative importance of these effects.

\section{Eddy damping}

Vortex shedding from the bilge corners is expected to be the most significant viscous effect. We apply the Pressure Damping Model with the pressure distribution in the gap related to the eddy damping discussed in section 3.5.2. The vortex induced damping effect on the piston-like elevation in the gap is displayed in figure 6.20 for some given values of the heave amplitude. Far-field amplitudes are shown in figure 6.21. Both amplitudes has been reduced due to the simulated eddy damping. The accompanying added mass and damping coefficient are displayed in figure 6.23 and 6.24 .

We introduce the term relative damping and define it as

$$
\frac{\Delta A_{g}}{A_{g 0}}=\frac{A_{g 0}-A_{g}}{A_{g 0}},
$$

where $A_{g 0}$ is the amplitude when no additional damping is applied, and $A_{g}$ is the damped amplitude. The relative damping is then describing the proportion of reduction in amplitude, relative to the original amplitude. Figure 6.22 displays the relative damping for three different gap lengths, for a continuous variation of the heave amplitude. Note from the figure that the relative damping does not have $\Delta A_{g} / A_{g 0}=1$ as a limit when the heave amplitude increases. When the relative damping exceeds 1 , the pressure generated wave contribution is larger than the original. The result will be a wave with opposite phase of the original wave. This is of course completely unphysical, and a consequence of the formulation. This suggests that the method is only valid for a range of heave amplitudes. As discussed in section 6.3.1, applied pressure which result in a local minimum at resonance is questionable. The dashed lines in figure 6.22 indicates where the results tends to exhibit this behaviour. 


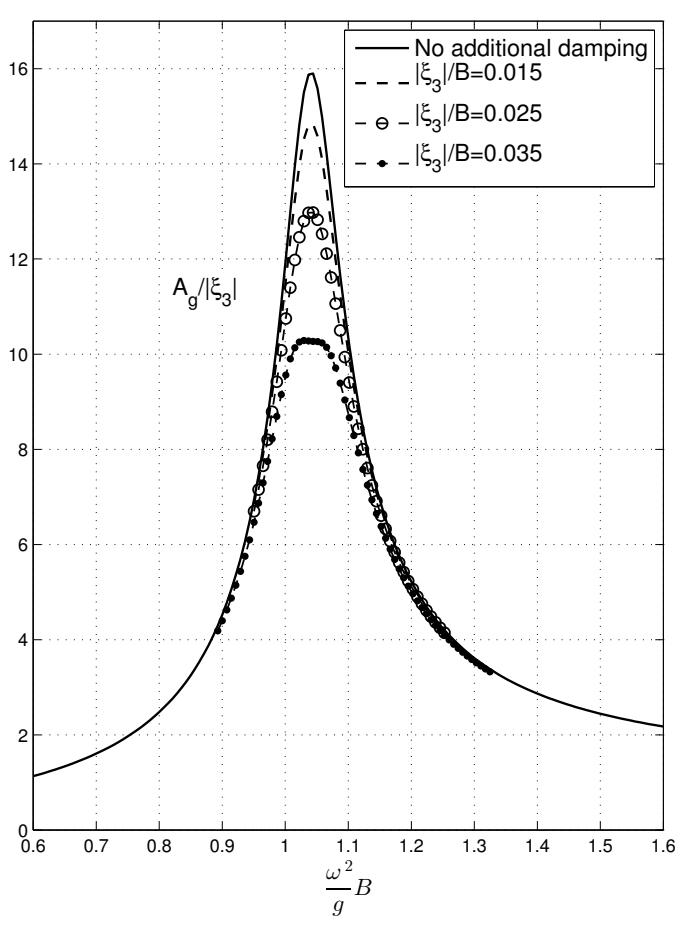

Figure 6.20: Non-dimensional gap amplitude $A_{g} /\left|\xi_{3}\right|$. $B / D=2, b / D=1$.

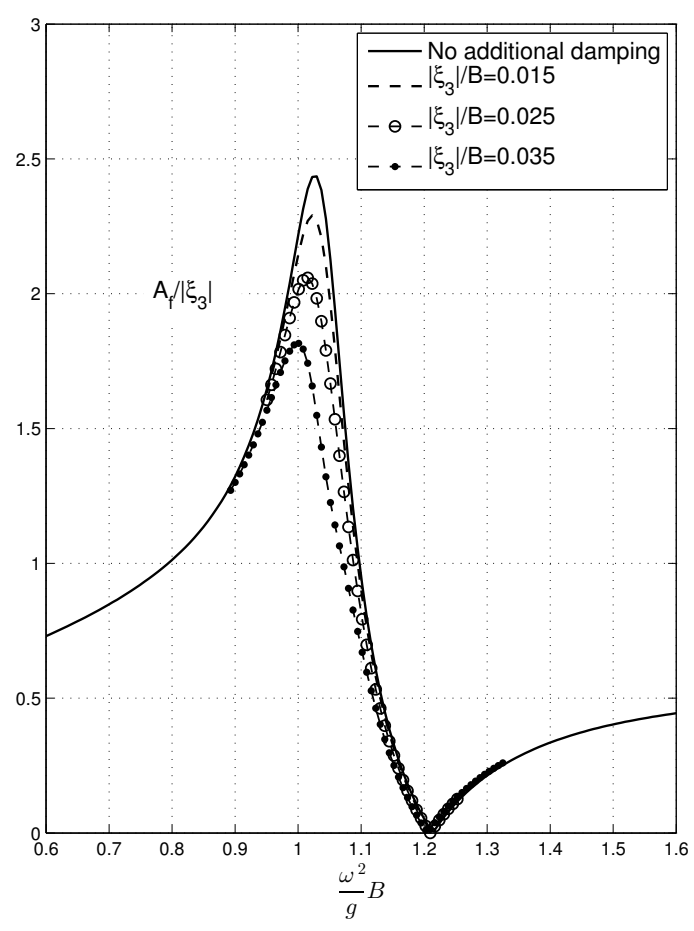

Figure 6.21: Non-dimensional far-field amplitude $A_{f} /\left|\xi_{3}\right| . B / D=2, b / D=1$.

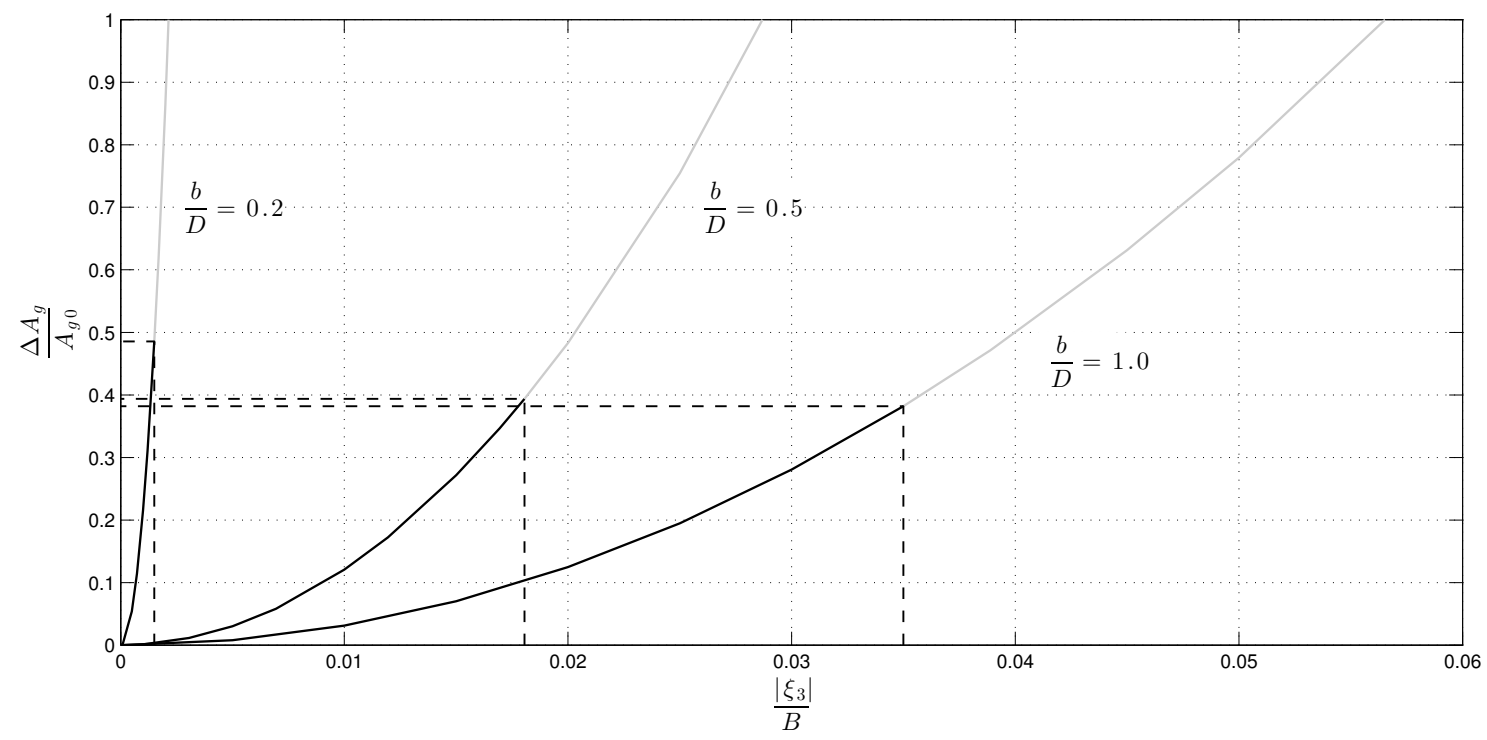

Figure 6.22: Relative damping for three different gap lengths for various values of the heave amplitude. $B / D=2$. 


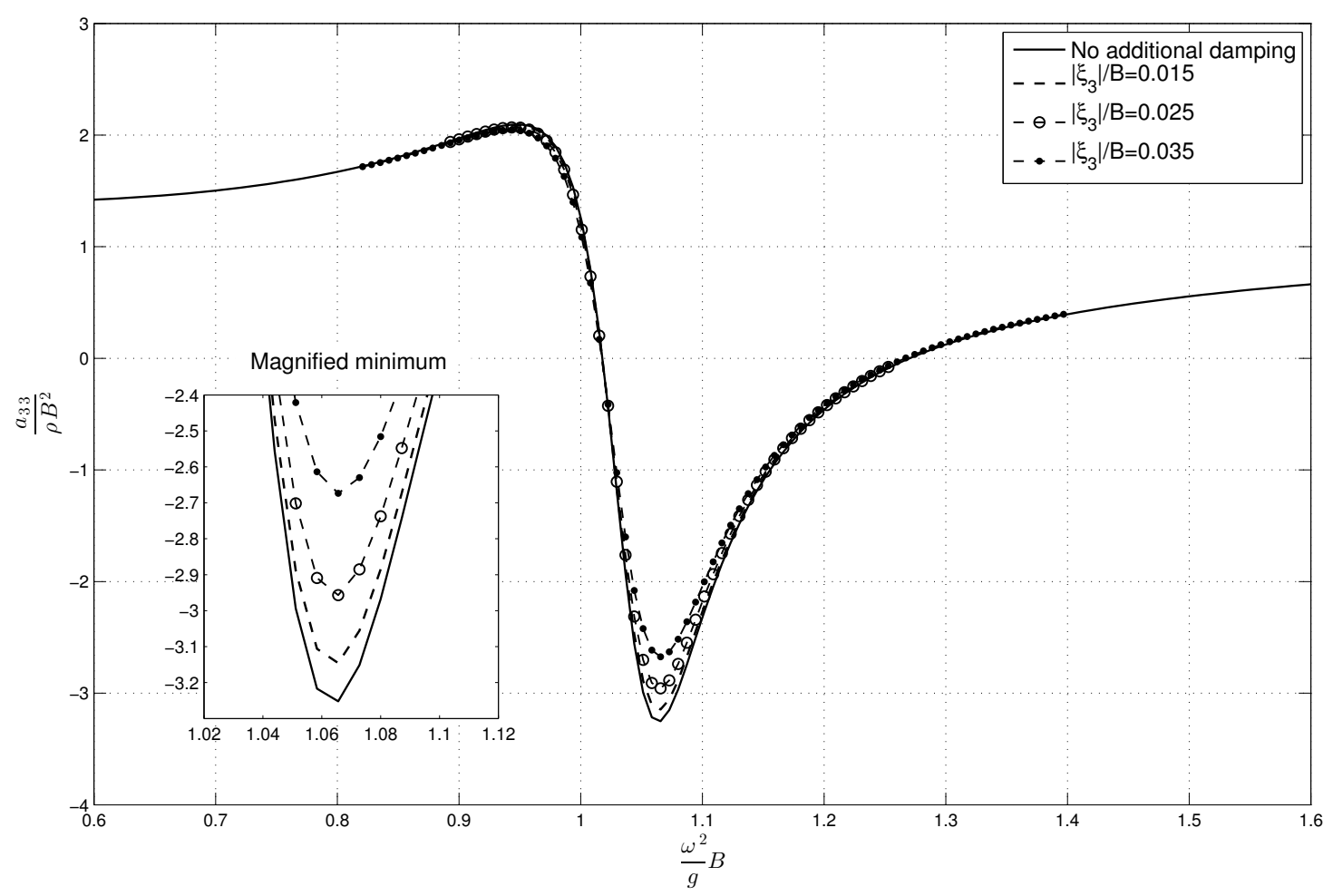

Figure 6.23: Non-dimensional added mass for different heave amplitudes. $B / D=2, b / D=1$.

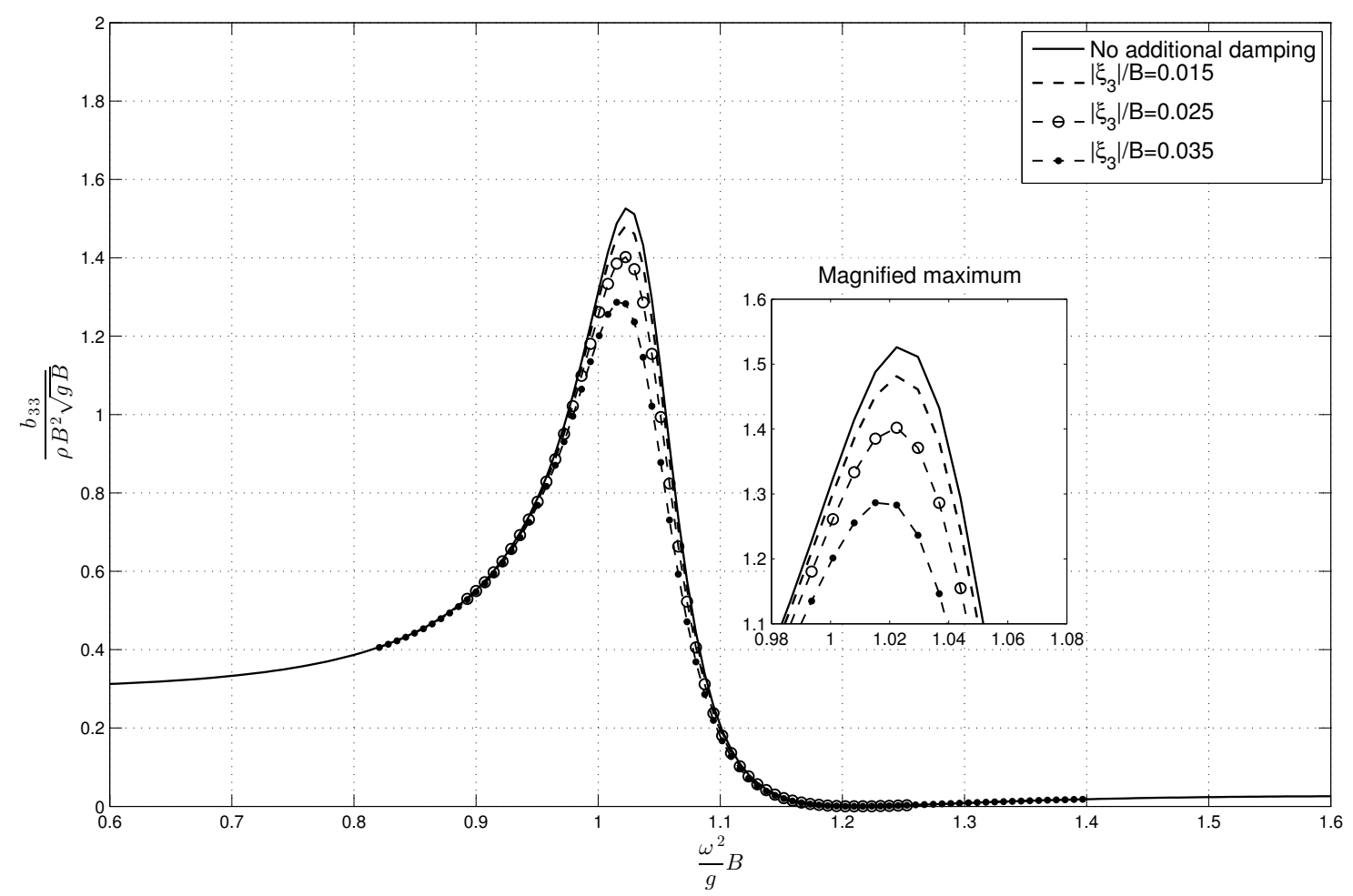

Figure 6.24: Non-dimensional damping coefficient for different heave amplitudes. $B / D=2, b / D=1$. 
The pressure will do work on the free surface. We can calculate the work done by the pressure over one cycle as

$$
\bar{W}=-\overline{F v},
$$

where $v$ is the free surface velocity. The energy will be transported away with the pressure generated waves. We may controll the calculations by the the energy flux in the far-field given in (6.5). Figure 6.25 displays both the average work and the average energy flux for three different heave amplitudes. The pressure force is dependent on the wave amplitude squared, and hence the work gets a cubic dependence. As the pressure force represents the viscous force, and it does work against the free surface motion this could be regarded as energy dissipated by viscousity.

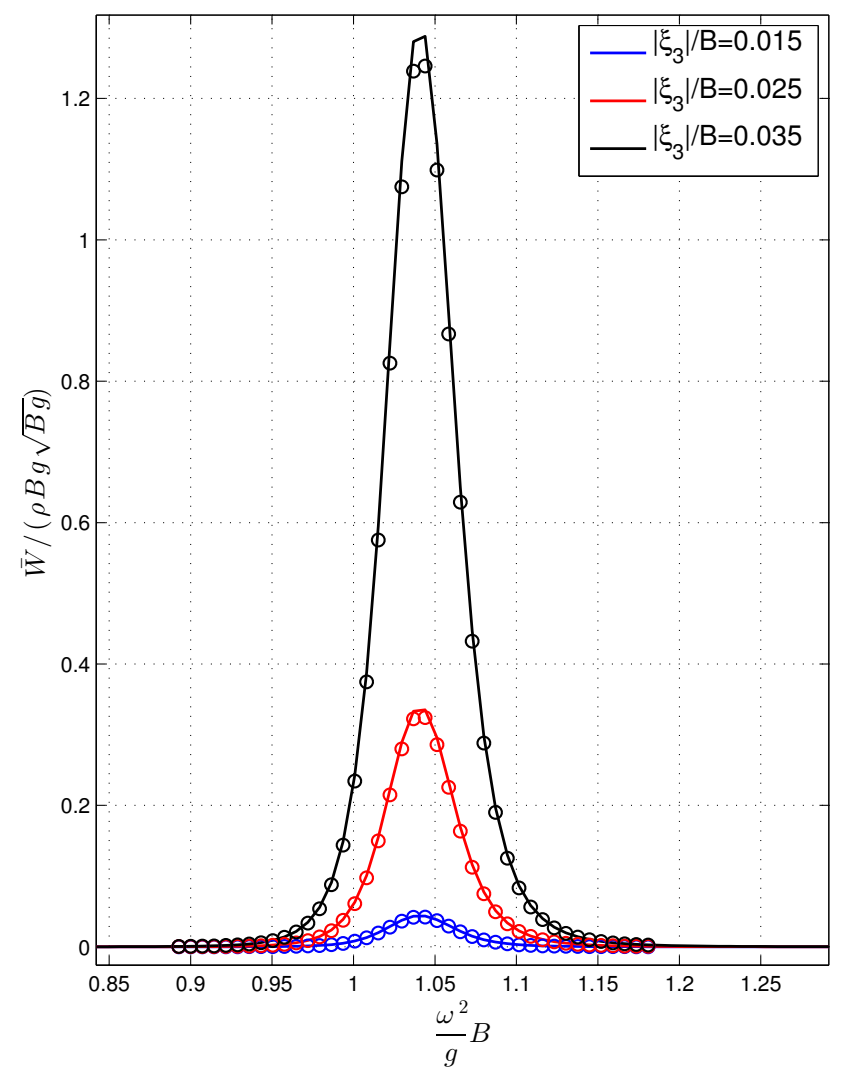

Figure 6.25: Average work done by the free surface pressure in the gap. The circles indicates calculated values for the average far-field energy flux. $B / D=2$, $b / D=1$. 


\section{Skin friction}

The skin friction effects on the gap wave amplitude is considered. The same forcing amplitudes as in figure 6.20 are considered to get an impression of the relative importance. Figure 6.26 displays the reduction in wave amplitude around the gap resonance frequency. It is evident that this effect is negligible compared to effect of vortex shedding. This also implies that the effect of skin friction has negligible impact on the hydrodynamic coefficients. Skin friction will not be considered further.

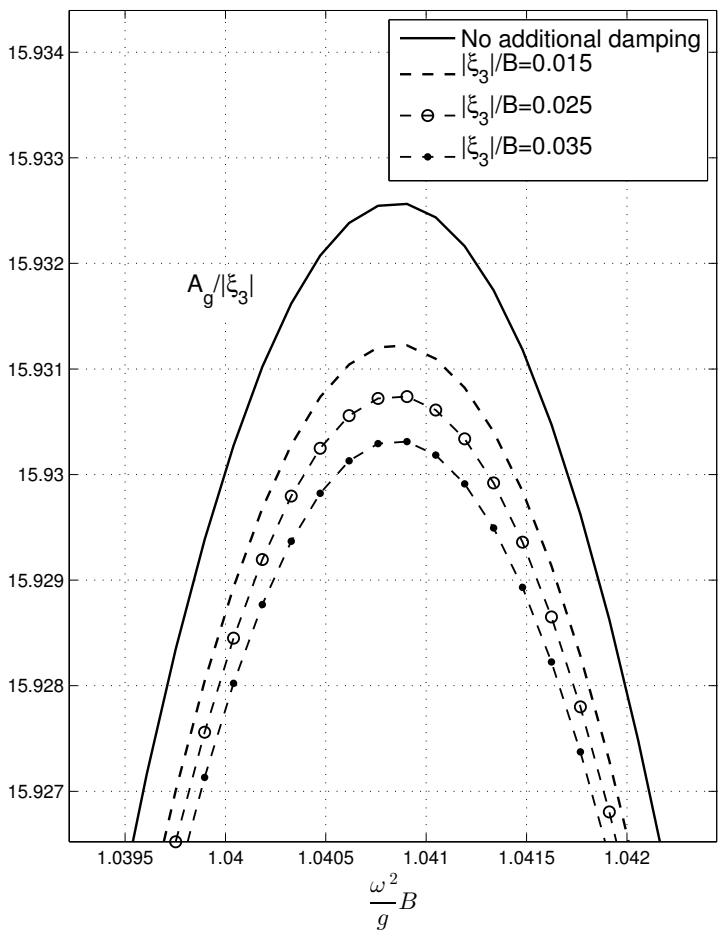

Figure 6.26: Effect of skin friction on the piston mode amplitude around the gap resonance frequency. The effect is negligible compared to the effect of vortex shedding. Note the scale on the y-axis. $B / D=2, b / D=1$. 


\section{Bilge keel effects}

The viscous effect associated with bilge keels is considered. This will ultimately be an additional effect which adds up to the eddy damping, but here we consider the individual effect. Two different widths has been tested. Figure 6.27a and $6.27 \mathrm{~b}$ shows the reduction in amplitude due to bilge keels with width $B_{b k} / b=0.1$ and $B_{b k}=0.2$, respectively. The length of one keel is $5 \%$ of the body width $B$ in the first case, and $10 \%$ for the second. Although more apparent for the larger bilge keels, the effect is small compared to the eddy damping.

The pair of bilge keels covers the gap opening by $20 \%$ in the first case, and $40 \%$ in the second case. This should result in less fluid entering the gap. This has not been accounted for, as only the viscous contribution from the bilge keels has been considered. The effect of covering the gap is presumably a potential flow effect, and could be captured by modeling the bilge keels in the panel model.

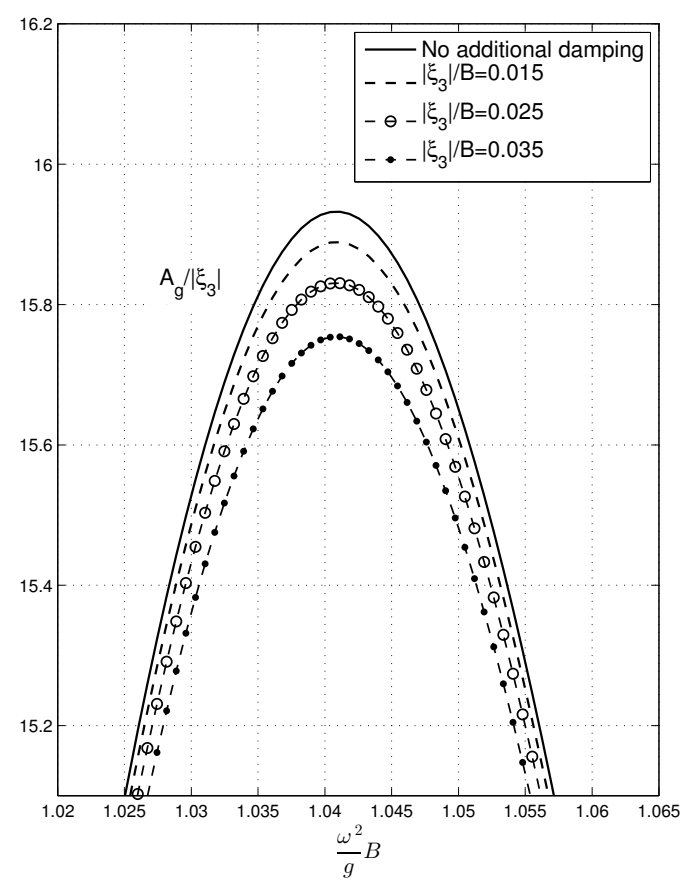

(a) Bilge keel width $B_{b k} / b=0.1$. $B / D=2, b / D=1$

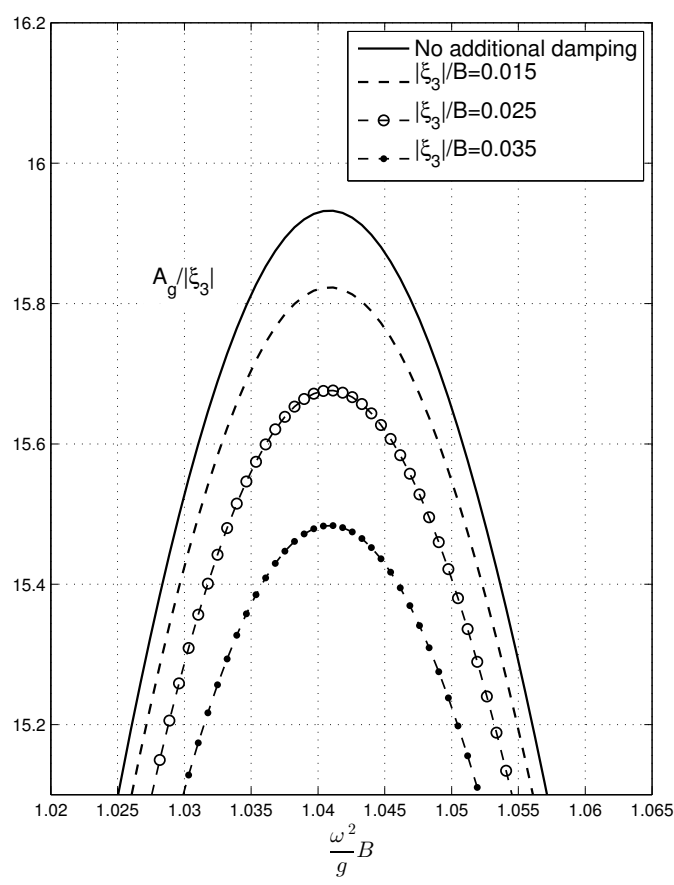

(b) Bilge keel width $B_{b k} / b=0.2$. $B / D=2, b / D=1$

Figure 6.27: Non-dimensional gap amplitude. Viscous effect from bilge keels are considered. 


\subsection{Newtonian Cooling Damping Model}

The newtonian cooling damping model is applied in the gap. Forced heave motion is considered to obtain comparable results to the Pressure Damping Model in 6.3. We give the damping paramter $\nu$ different values to obtain different degree of damped elevation and hydrodynamic coefficients.

\subsubsection{Heaving of rectangular boxes}

The newtonian cooling damping model is not dependent on the value of the heave amplitude $\xi_{3}$. The resulting damping depends only on the damping coefficient $\nu$ for a given geometry. Figure 6.28 displays the gap wave amplitude. Figure 6.29 displays the associated far-field aplitudes. A noteworthy occurrence in the far-field takes place around $\omega^{2} B / g=1.2$, where the basis solution has its minimum. For the consecutive frequencies, the amplitudes is larger than for the basis solution. The reason for this is clearfied by the damping coefficient. The added mass and damping coefficient are displayed in 6.31 and 6.32. We observe that the overall resonant behaviour is broaden out for increasing values of $\nu$. This results in larger damping coefficients in the frequency region after resonance. The damping is directly related to the far-field waves and hence larger amplitude is expected. Not much is to say about the Newtonian cooling effect on the gap wave amplitude, as well as the added mass coefficient. Their magnitudes are reduced for increasing values of $\nu$, as expected.

The relative damping in the gap is displayd in 6.30 , for three different gap widths. The relative damping has asymptotes $\Delta A_{g} / A_{g 0}=1$. This implies that the wave elevation will eventually be damped to zero for larger values of $\nu$. We also observes that a smaller value of $d / B$ leads to larger relative damping for any given $\nu$.

\section{Comparison with Wasim}

The Newtonian cooling formulation is originally included in Wasim for use in numerical beach context. The formulation may also be applied on areas of the free surface which is not a part of the numerical beach. Wasim has been used with Newtonian cooling in the gap to obtain damped gap wave amplitudes for the situation discussed in 6.2.4. We compare them to result from our present $2 \mathrm{D}$ code. As discussed in $6.2 .4,2 \mathrm{D}$ and $3 \mathrm{D}$ results are not directly comparable mainly due the variation in elevation in the length direction of the gap in 3D. Nevertheless, the peak wave amplitude is of approximately equal value and we will see how this is affected by the Newtonian cooling damping for some given $\nu$-values. The result is shown in figure 6.33 . We observe that the $3 \mathrm{D}$ calculations seems to be slightly more affected by the Newtonian cooling. 


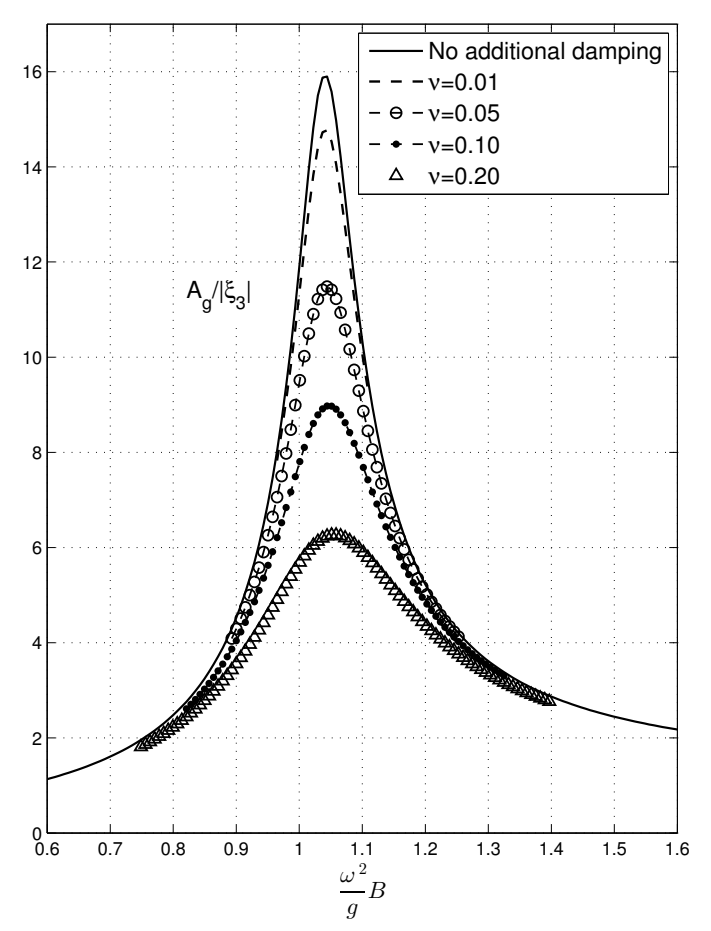

Figure 6.28: Non-dimensional gap amplitude.

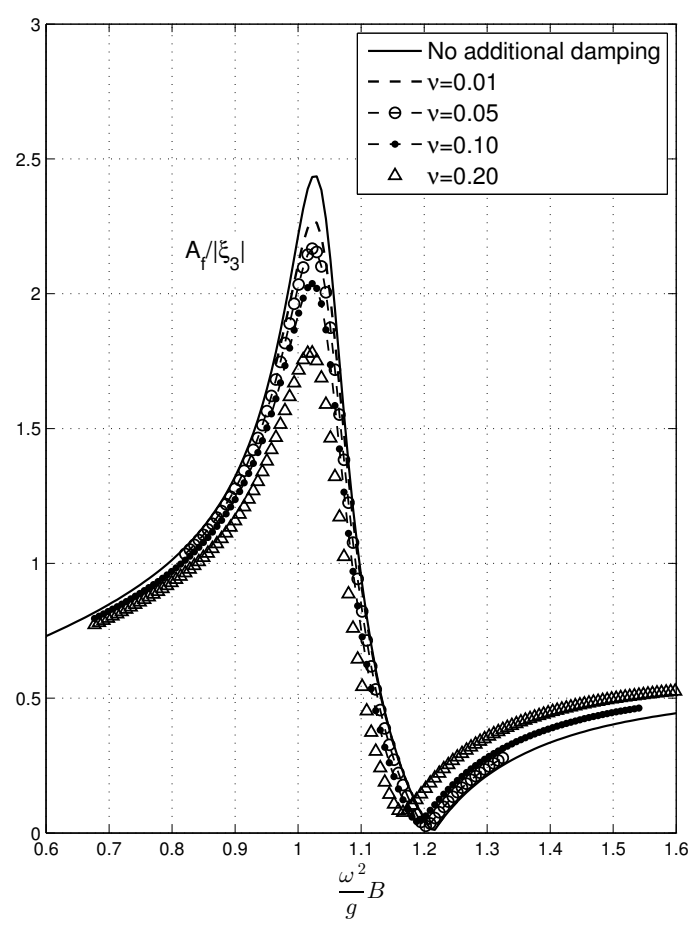

Figure 6.29: Non-dimensional far-field amplitude.

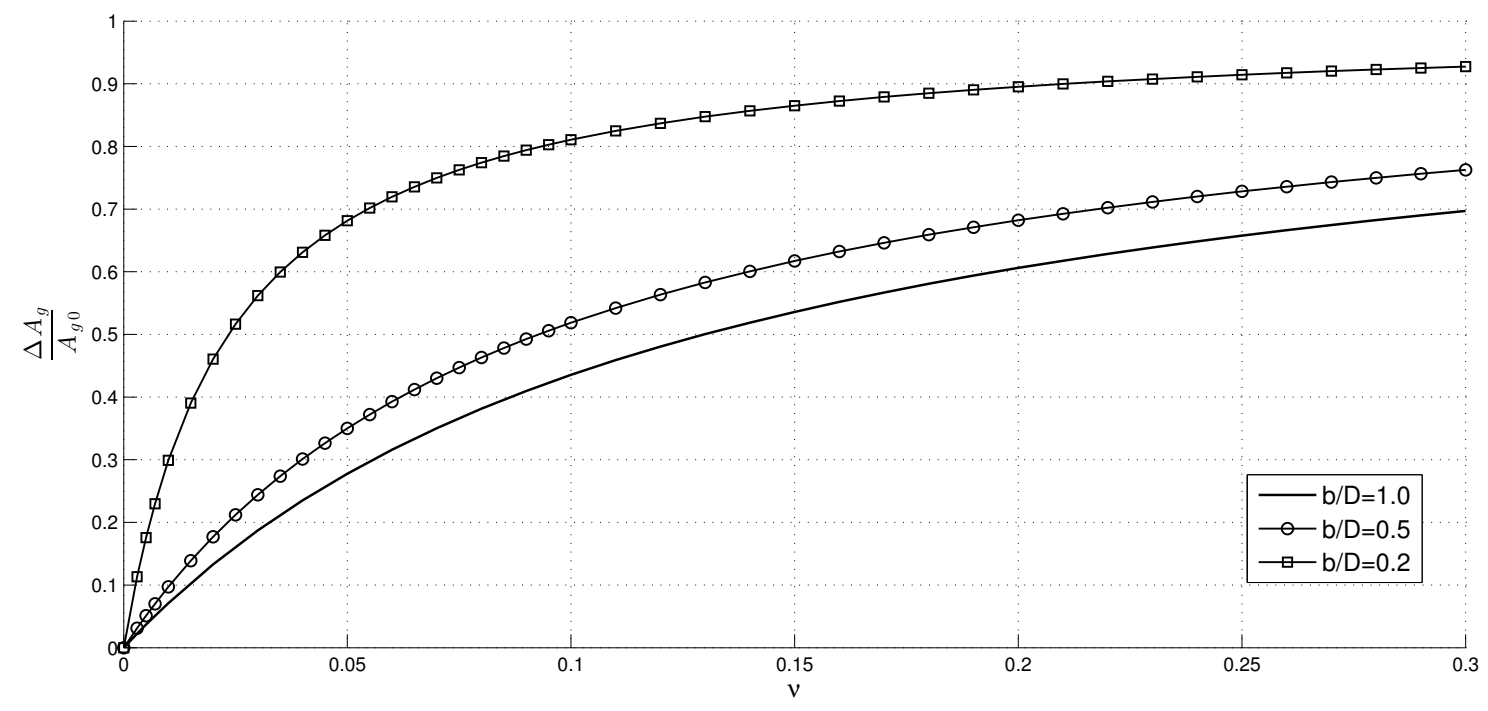

Figure 6.30: Relative damping for three different gap lengths for various values of the damping coefficient. $B / D=2$ 


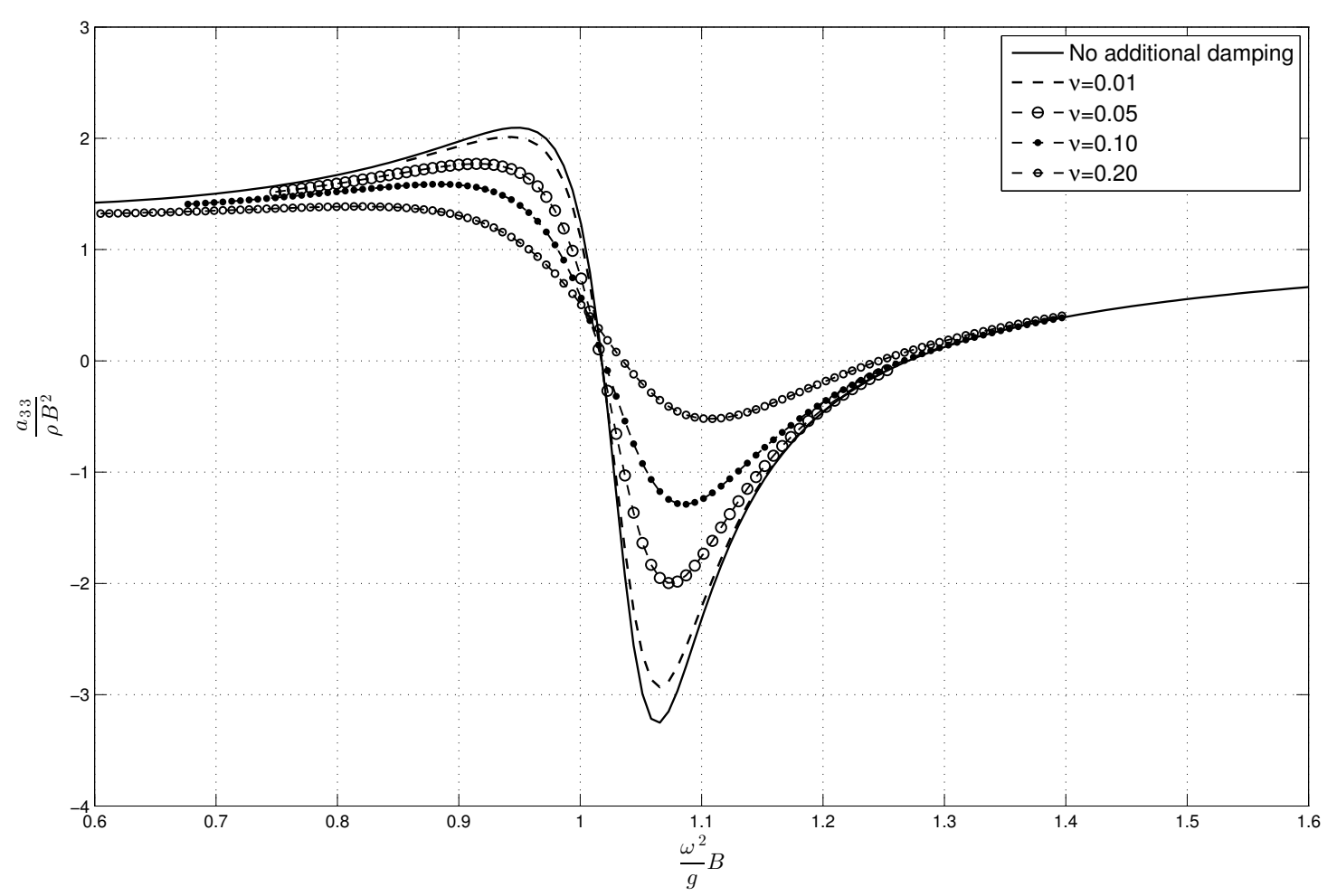

Figure 6.31: Non-dimensional added mass for different values of $\nu . B / D=2, b / D=1$.

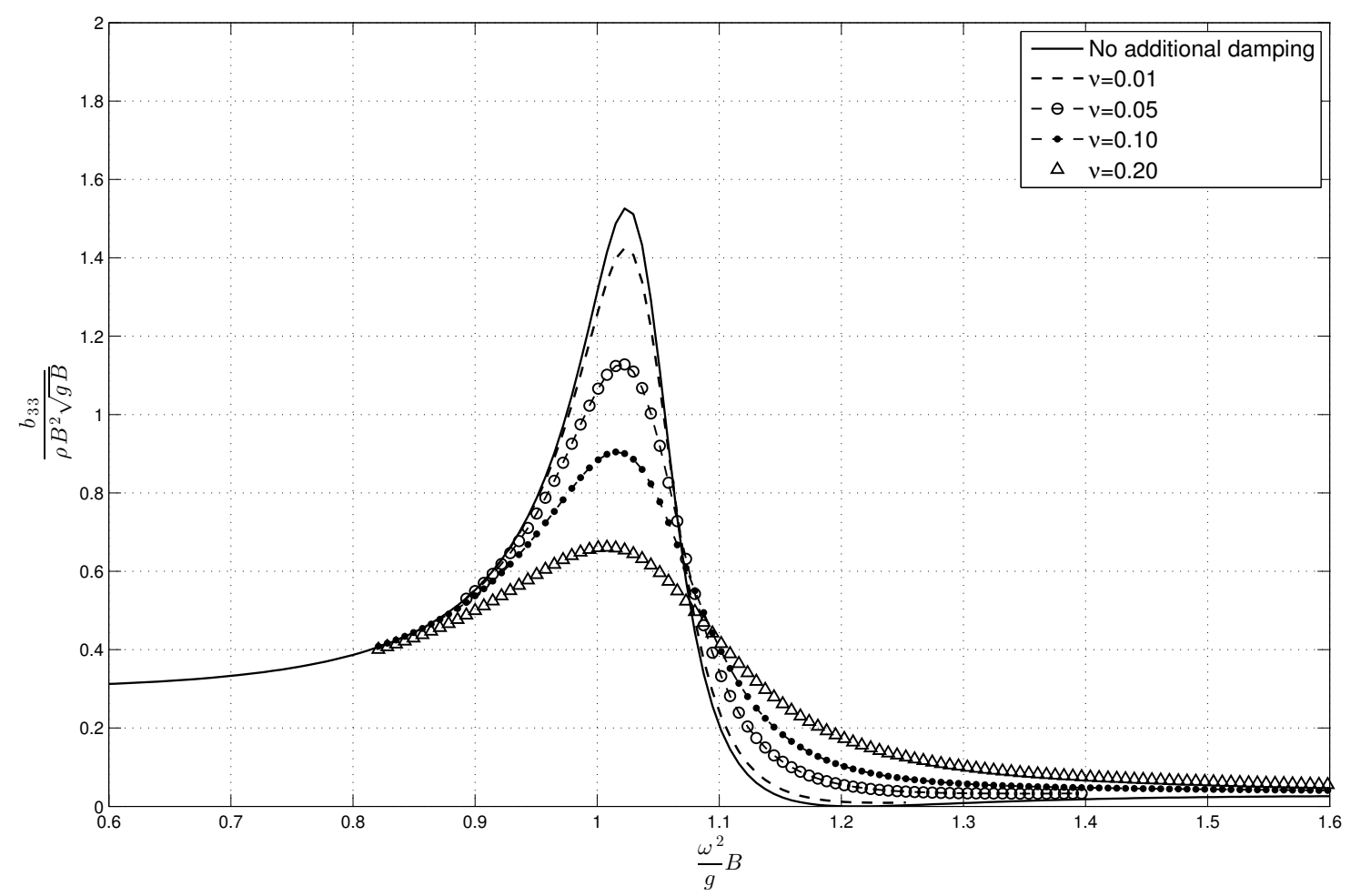

Figure 6.32: Non-dimensional damping coefficient for different values of $\nu . B / D=2, b / D=1$. 


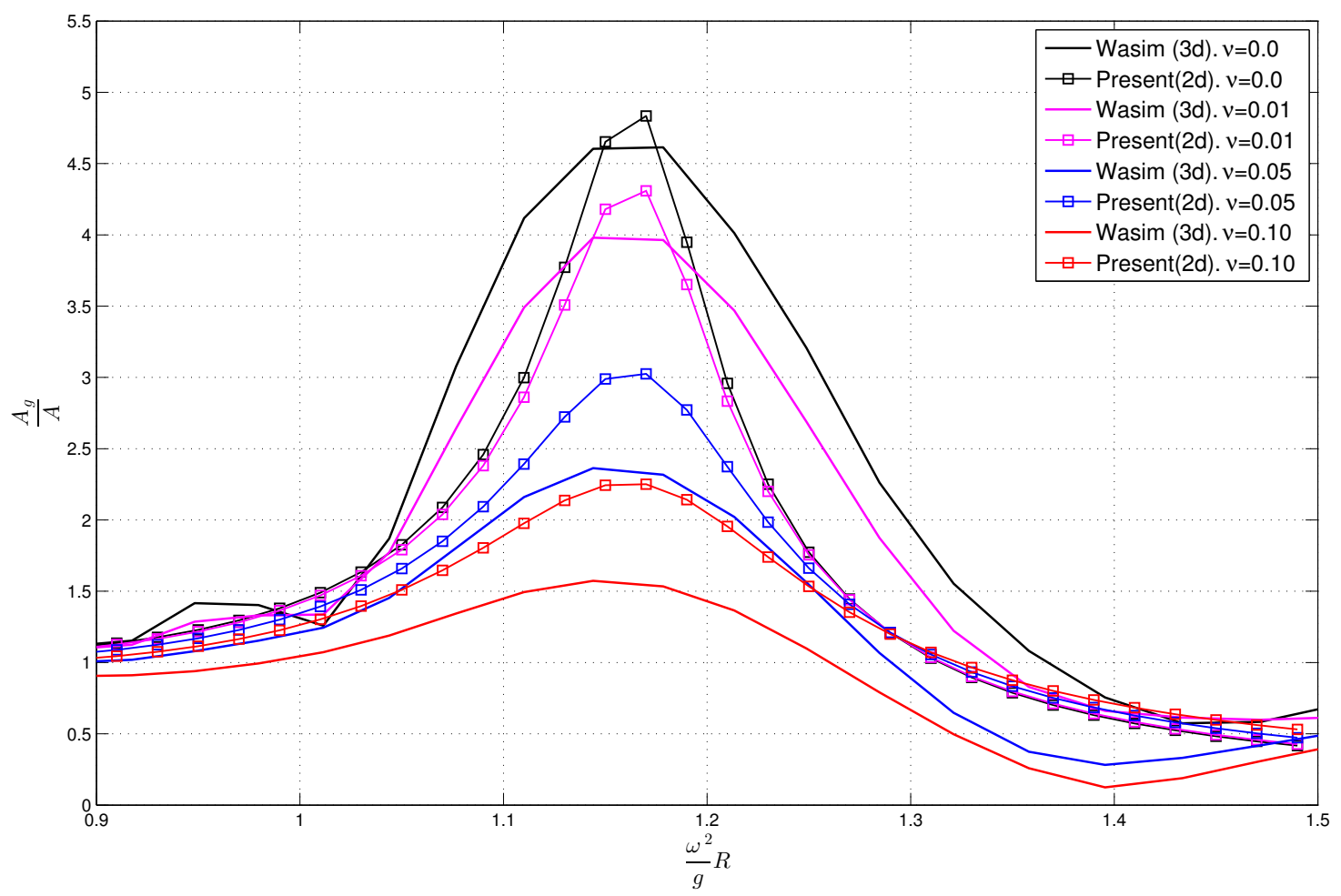

Figure 6.33: Wasim(3D) with Newtonian cooling, compared to present(2D) results. Circular cylinders as in section 6.2.4 


\subsection{Comparison of the damping models}

We have demonstrated how both the Pressure Damping Method and the Newtonian Cooling Damping Model provides damping of the heave induced piston-like motion in the gap. In the Pressure Damping Model we get larger damping effect when the forced motion amplitude is increased. This is due to the formelation of the pressure distribution (4.5), which is non-linear in the undamped wave amplitude. The Newtonian Cooling Model is linear and thus not affected by the amplitude of heave motion. Instead we may obtain different degree of damping by varying the damping coefficient $\nu$. It should be repeated that the value of $\nu$ is not related to physics, while the damping effect obtained for a given heave amplitude is related to the physical viscous effects. The viscous effects are non-linear, and so it seems questionable to model these effects by the linear Newtonian Cooling Damping Model, unless $\nu$ is somehow related to $\xi_{3}$.

Some key results obtained from the analysis in 6.4 and 6.3 are listed in table 6.1 and 6.2. For each $\left|\xi_{3}\right| / B$ there is a $\nu$ which gives the same damped amplitde at resonance. As an example we have shown calculation with $\left|\xi_{3}\right| / B=0.031$ and $\nu=0.057$ for $b / D=1.0$ in figure 6.34 . We see that the curves coincides well for all displayed frequencies. The reason for the minor discrepancy at the peak is that the values in table 6.1 are rounded.

\begin{tabular}{cccc}
\hline \multicolumn{4}{c}{ Relative damping (\%) } \\
\hline $10 \%$ & $20 \%$ & $30 \%$ & $40 \%$ \\
\hline$\frac{\left|\xi_{3}\right|}{B}=0.018$ & $\frac{\left|\xi_{3}\right|}{B}=0.025$ & $\frac{\left|\xi_{3}\right|}{B}=0.031$ & $\frac{\left|\xi_{3}\right|}{B}=0.037$ \\
$\nu=0.015$ & $\nu=0.034$ & $\nu=0.058$ & $\nu=0.080$ \\
\hline
\end{tabular}

Table 6.1: Comparison of the Newtonian cooling damping model and the pressure damping model. $b / D=1.0, B / D=2$

\begin{tabular}{cccc}
\hline \multicolumn{4}{c}{ Relative damping (\%) } \\
\hline $10 \%$ & $20 \%$ & $30 \%$ & $40 \%$ \\
\hline$\frac{\left|\xi_{3}\right|}{B}=0.009$ & $\frac{\left|\xi_{3}\right|}{B}=0.013$ & $\frac{\left|\xi_{3}\right|}{B}=0.016$ & $\frac{\left|\xi_{3}\right|}{B}=0.017$ \\
$\nu=0.012$ & $\nu=0.025$ & $\nu=0.045$ & $\nu=0.060$ \\
\hline
\end{tabular}

Table 6.2: Comparison of the Newtonian cooling damping model and the pressure damping model. $b / D=0.5, B / D=2$ 


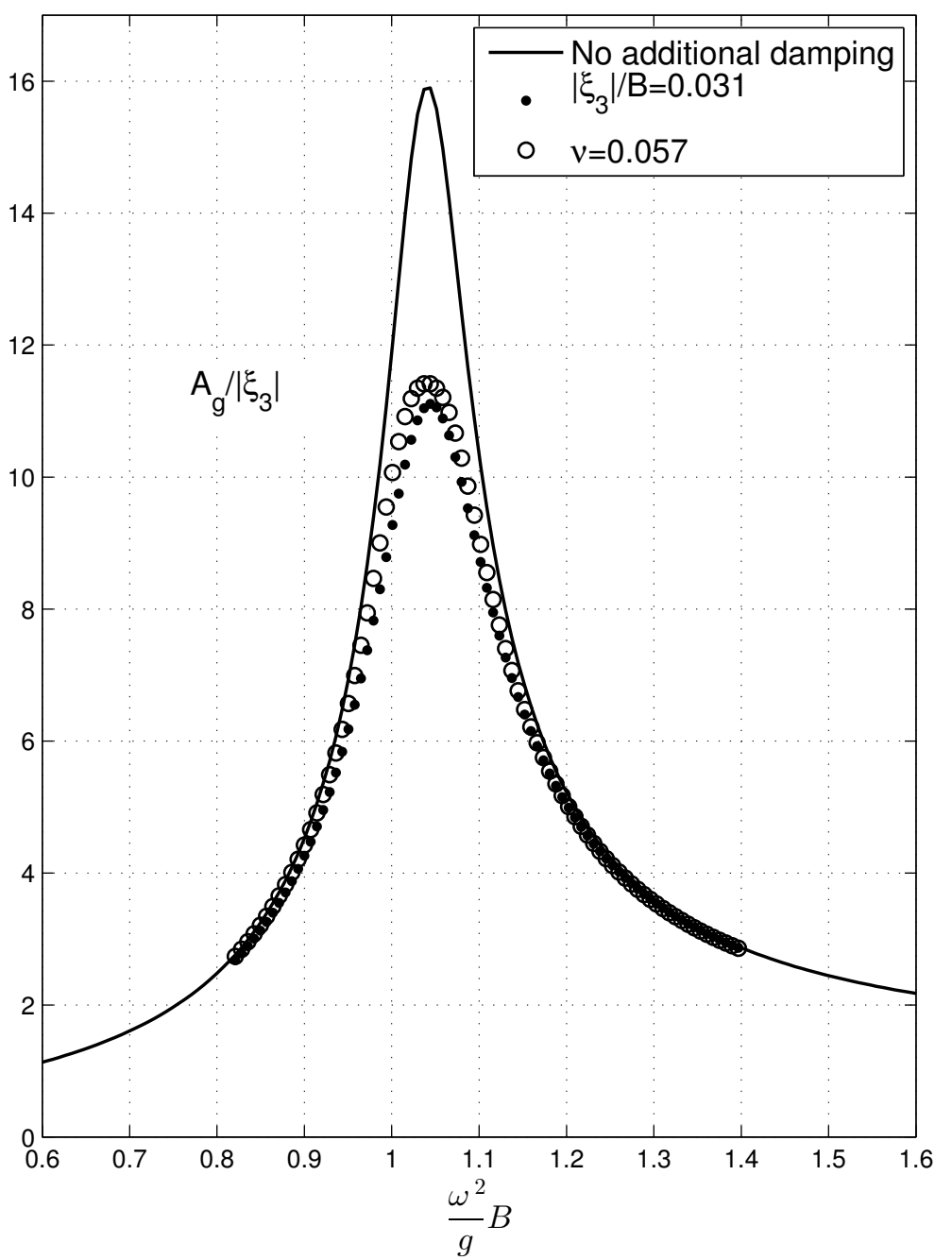

Figure 6.34: Comparison of the heave generated elevation damped by the two different damping models. The relative damping(\%) is $30 \%$. $b / D=1, B / D=2$ 


\section{Chapter 7}

\section{Summary and conclusion}

The resonant behaviour occuring when a gap is present has been numerically investigated with Boundary Element Method. Empirical formulas for the damping effect of different viscous forces were related to the Pressure Damping Model. By comparing the effect of skin friction and vortex shedding, we conclude that skin friction is negligible. Viscous effects from bilge keels in the gap were shown to be small compared to the eddy damping. However, the coverage of the gap by the keels were not considered. It is presumable that this is an important effect of bilge keels as they prevents as much water to enter the gap. Although some assumptions were made to form simple expressions for the viscous damping, we hope to have captured the leading effects. The procedure of relating the viscous forces to a pressure distribution on the gap surface is general, so any empirical formulas may be used. We have demonstraded how the Newtonian Cooling Damping Model can be compared to the Pressure Damping Model to yield estimates for the Newtonian cooling damping coefficient. If one are able to get good estimations for the viscous forces, this method could be a simple alternative to CFD analysis or model tests.

A three dimensional analysis was carried out using Wasim. In this context threedimensional flow effects were briefly discussed. We conclude that the analogy between three-dimensional cylinders and a two-dimensional cross section should not be pushed to far, as longitudinal variations occur in the gap in the threedimensional case. 
Appendices 


\section{Appendix A}

\section{Greens theorem}

Given the functions $f$ and $g$ defined in $\Omega$, bounded by the boundary $\delta \Omega$. Greens second identity relate the volume integral to a surface integral,

$$
\int_{\Omega}\left(f \nabla^{2} g-g \nabla^{2} f\right) \mathrm{d} V=\int_{\delta \Omega}\left(f \frac{\partial g}{\partial n}-g \frac{\partial f}{\partial n}\right) \mathrm{d} S .
$$

We consider two Laplacian potentials, $\varphi$ and $\psi$ defined in the volume $V$, with boundary $S$. It follows from Greens theorem that.

$$
\int_{S}\left(\varphi \frac{\partial \psi}{\partial n}-\psi \frac{\partial \varphi}{\partial n}\right) \mathrm{d} S=0
$$

We let $\psi$ be a source with fieldpoint $\mathbf{x}$ and source point $\boldsymbol{\xi}$. The two dimensional source

$$
\psi=\ln r
$$

where $r=|\mathbf{x}-\boldsymbol{\xi}|=\sqrt{(x-\xi)^{2}+(z-\zeta)}$. When $r=0$ then $\psi$ becomes singular, and does not satify $\nabla^{2} \psi=0$. Except at the singular point the function is Laplacian. We enclose the singular point $\mathbf{x}-\boldsymbol{\xi}$ by a circle or half circle if $\mathbf{x}$ is on the fluid boundary. The contributions from the limits of the circle/halfcircle around the singularity could be found by taylor-expansion. The resulting expression is

$$
\int_{S}\left(\varphi(\boldsymbol{\xi}) \frac{\partial G(\boldsymbol{\xi} ; \mathbf{x})}{\partial n_{\boldsymbol{\xi}}}-G(\boldsymbol{\xi} ; \mathbf{x}) \frac{\partial \varphi(\boldsymbol{\xi})}{\partial n_{\xi}}\right) \mathrm{d} \boldsymbol{\xi}= \begin{cases}0, & \text { outside the fluid domain } \\ \pi \varphi(\mathbf{x}), & \text { on the boundary } \\ 2 \pi \varphi(\mathbf{x}), & \text { inside the fluid domain }\end{cases}
$$




\section{Appendix B}

\section{Equivalent linearization}

Consider a non-linear force given by

$$
F=\frac{1}{2} \rho L_{c}^{2} C_{d}|v| v
$$

as in 4.2.1, where $v=v(t)=V_{0} \cos (\omega t+\theta)$. The phase $\theta$ may be disregarded for this derivation. We denote the constants as $F_{0}=1 / 2 \rho L_{c}^{2} C_{d}$ for simplicity. We seek to find a linear expression on the form

$$
\hat{F}=K F_{0} v,
$$

where $K$ is an unknown constant, so that the work done over one period is the same for this expression and the non-linear expression.

The work done by $F$ over one period is

$$
\begin{aligned}
W & =\int_{0}^{2 \pi / \omega} F_{0} V_{0}^{2} \cos (\omega t)|\cos (\omega t)| \cdot V_{0} \cos (\omega t) \mathrm{d} t \\
& =F_{0} \frac{8 V_{0}^{3}}{3 \omega} .
\end{aligned}
$$

The work done by $\hat{F}$ over one period is

$$
\begin{aligned}
\hat{W} & =\int_{0}^{2 \pi / \omega} K F_{0} V_{0} \cos (\omega t) \cdot V_{0} \cos (\omega t) \mathrm{d} t \\
& =K F_{0} \frac{\pi}{\omega} V_{0}^{2}
\end{aligned}
$$

The constant $K$ is found by equation $\hat{W}$ and $W$,

$$
\begin{aligned}
& K F_{0} \frac{\pi}{\omega} V_{0}^{2}=F_{0} \frac{8 V_{0}^{3}}{3 \omega} \\
& K=\frac{8 V_{0}}{3 \pi}
\end{aligned}
$$

The equivalent linearized expression for $F$ is now given as

$$
\begin{aligned}
F \approx \hat{F} & =\frac{8}{3 \pi} F_{0} V_{0} v \\
& =\frac{4}{3 \pi} \rho L_{c}^{2} C_{d} V_{0} v .
\end{aligned}
$$




\section{Appendix C}

\section{Convergence test}

Convergence of the computed piston mode amplitude is considered. The result is displayed in figure C.1. The percentage difference for the peak amplitude between $N=54$ and $N=78$ is $1.065 \%$. The percentage difference betweem the frequencies where peak amplitude occurs for $N=54$ and $N=78$ is $0.192 \%$. The percentage difference for the peak amplitude between $N=72$ and $N=78$ is $0.18 \%$. A number of $N=72$ panels where used throughout the numerical study in chapter 6. Percentage difference bewteen two value is given as

$$
\Delta_{\text {percentage }}=\frac{\left|x_{1}-x_{2}\right|}{\left(x_{1}-x_{2}\right) / 2},
$$

where $x_{1}$ and $x_{2}$ represents the two values.

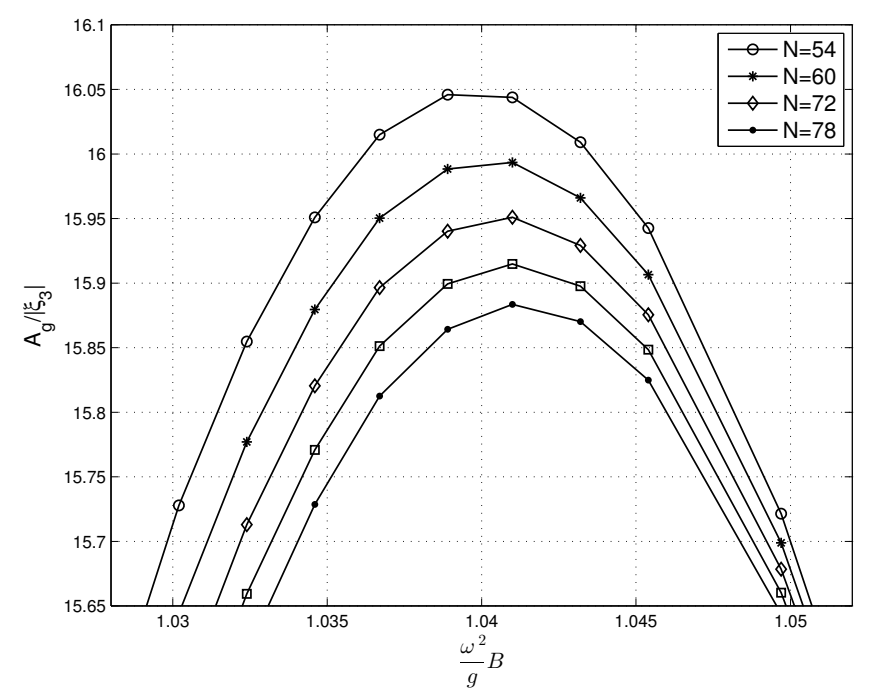

Figure C.1: Results from convergence test. Nondimensional piston mode amplitude $A_{g} /\left|\xi_{3}\right|$ for different number of panels. Note the axes. 


\section{Appendix D}

\section{Equation of motion}

Motions in two dimensions are considered, with $i, j=2,3,4$ representing sway, heave and roll respectively. The equation of motion for a freely floating body follows from Newton's second law,

$$
F_{i}=\sum_{j=2}^{4} M_{i j} \dot{U}_{j} \quad i, j=2,3,4 .
$$

The body acceleration is given as

$$
\dot{U}_{j}=\operatorname{Re}\left\{-\omega^{2} \xi_{j} e^{i \omega t}\right\} \quad i, j=2,3,4 .
$$

The product $M_{i j} \dot{U}_{j}$ is the body inertia forces. The mass matrix $M_{i j}$ for our two-dimensional problem is given as

$$
M_{i j}=\left(\begin{array}{ccc}
m & 0 & 0 \\
0 & m & 0 \\
0 & 0 & I_{11}
\end{array}\right)
$$

with the body mass as the displaced water volume times the density

$$
m=\rho V
$$

and the roll moment of inertia

$$
I_{11}=\iint \rho_{B}\left(\mathbf{x} \cdot \mathbf{x}-x_{1}^{2}\right) \mathrm{d} V
$$

The inertia forces equals the forces which acting on the body. We have defined the force due to body motion in (6.1), and the force from the incident wave in (6.7). In addition to these force components we have the restoring force. The restoring force is zero for sway motion, while heave and roll restoring force coefficients are given as

$$
\begin{aligned}
& c_{33}=\rho g S \\
& c_{44}=\rho g V\left[\frac{S_{22}}{V}+y_{B}-y_{G}\right]
\end{aligned}
$$


with $S_{22}$ as the waterplane moment, and $y_{B}$ and $y_{G}$ is the center of bouyancy and the center of gravity. The total restoring force is proportional to $\xi_{j}$. As all forces are varying with as $e^{i \omega t}$ we may omit the factor and express the equation of motion through the added mass and damping coefficient (6.3), restoring force coefficient (D.6) and the excitation force coefficients (6.8),

$$
\sum_{j=1}^{4} \xi_{j}\left(-\omega^{2}\left(M_{i j}+a_{i j}\right)+i \omega b_{i j}+c_{i j}\right)=A X_{i}
$$

for $i, j=2,3,4$ 


\section{Bibliography}

[1] Bearman P.W. Vortex Shedding From Oscillating Bluff-Bodies. Annual Review of Fluic Mechanics 1984;vol16:195-222

[2] Buchner B, Van Dijk A, De Wilde J. Numerical Multiple-Body Simulations of Side-by-Side Mooring to an FPSO. Proc. of IOPEC2001.

[3] Chen X., Duan W. Multi-domain Boundary Element Method with Dissipation. Journal of Marine Science and Applications 2012;11:18-23

[4] Faltinsen O.M. Resonant liquid motion in marine hydrodynamics. Procedia Engineering 2015;126:6-11

[5] Heo JK, Park JC, Koo WC, Kim MH. Influences of Vorticity to Vertical Motion of Two-Dimensional Moonpool under Forced Heave Motion. Mathematical Problems in Engineering Volume 2014

[6] Israeli M, Orszag S.A. Approximation of Radiation Boundary Conditions. Journal of Computational Physics 1981;41:115-135

[7] Kristiansen T, Faltinsen O.M. A two-dimensional numerical and experimental study of resonant coupled ship and piston-mode motion. Applied ocean Reasearch 2012;32:158-176

[8] Kristiansen T., Faltinsen O.M. Gap resonance analyzed by a domaindecomosition method combining potential and viscous flow DRAFT. Applied Ocean Research 2012;34:198-208

[9] Molin B. On the piston mode in moonpools. Proc. 14th Int. Workshop on Water Waves \& Floating Bodies Port Huron, 1999

[10] Newman J.N. Marine Hydrodynamics, MIT Press 1977.

[11] Newman J.N. Progress in wave load computations on offshore structures. Proc 23th OMAE, Vancouver, Canada, 2004.

[12] Lee, C.-H. and Nielsen, F.G Analysis of oscillating-water column device using a panel method., presented at 11th International Workshop on Water Waves and Floating Bodies, Hamburg, 1996

[13] Ogilvie TF. Oscillating Pressure Field on A Free Surface. 1969

[14] Sun L, Taylor P. First- and second order analysis of resonant waves between adjacent barges. Journal of Fluids and Structures 2010 
[15] Wang H, Falzarano JM. Energy extraction from the motion of an oscillating water column. Oscean System Engineering 2013;vol3:327-348

[16] Wehausen J.V, Laitone E. V. Surface waves. Handbuch der Physics 1960; Vol. 9 